U.S. Department of the Interior

U.S. Geological Survey

Prepared in cooperation with the

Massachusetts Department of Environmental Protection,

Division of Watershed Management, and Cape Cod Commission

\title{
Wetland Plants and Algae in a Coastal Marsh, Orleans, Cape Cod, Massachusetts
}

Water-Resources Investigations Report 98-4011

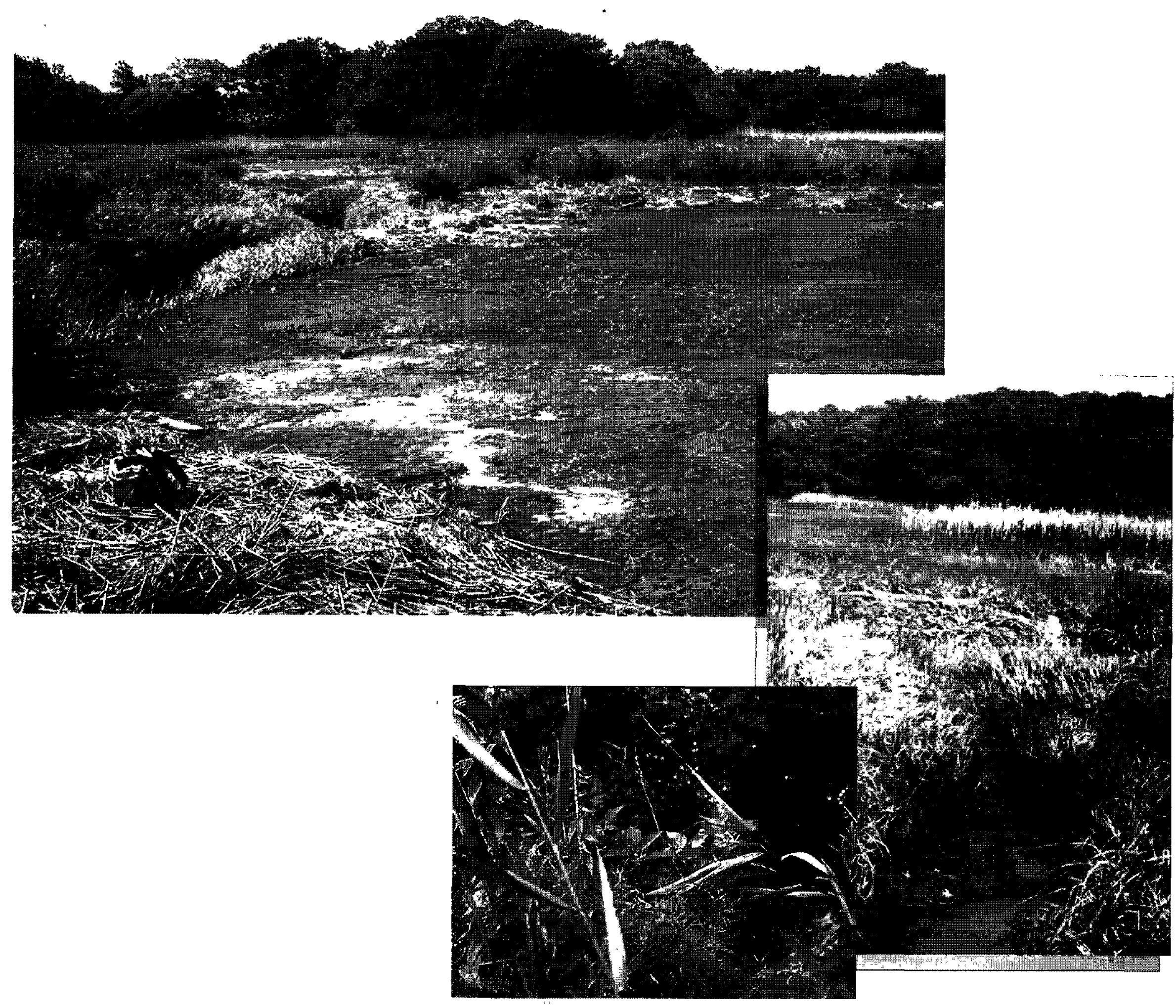




\section{Wetland Plants and Algae in a Coastal Marsh, Orleans, Cape Cod, Massachusetts}

By LESLIE A. DESIMONE, U.S. Geological Survey, BRIAN L. HOWES, DALE G. GOEHRINGER, Woods Hole Oceanographic Institution, and PETER K. WEISKEL, U.S. Geological Survey

U.S. Geological Survey

Water-Resources Investigations Report 98-4011

Prepared in cooperation with the

MASSACHUSETTS DEPARTMENT OF ENVIRONMENTAL PROTECTION, DIVISION OF WATERSHED MANAGEMENT and CAPE COD COMMISSION 


\title{
U.S. DEPARTMENT OF THE INTERIOR \\ BRUCE BABBITT, Secretary
}

\author{
U.S. GEOLOGICAL SURVEY
}

Thomas J. Casadevall, Acting Director

The use of trade or product names in this report is for identification purposes only and does not constitute endorsement by the

U.S. Geological Survey.

For additional information write to:

Chief, Massachusetts-Rhode Island District

U.S. Geological Survey

Water Resources Division

28 Lord Road, Suite 280

Marlborough, MA 01752
Copies of this report can be purchased from:

U.S. Geological Survey

Information Services

Box 25286, Federal Center

Denver, CO 80225-0286 


\section{CONTENTS}

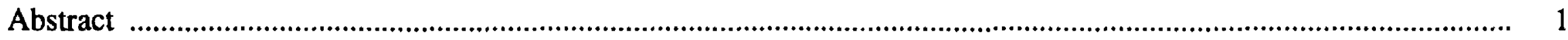

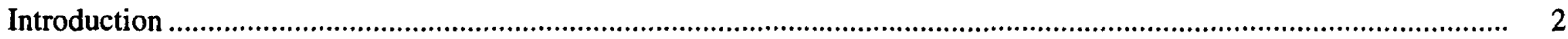



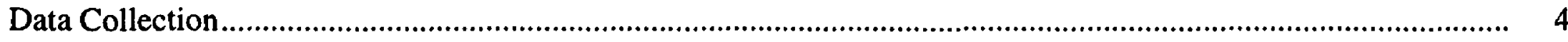

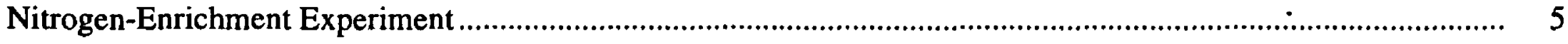

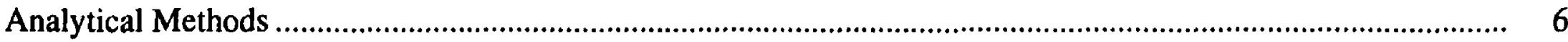

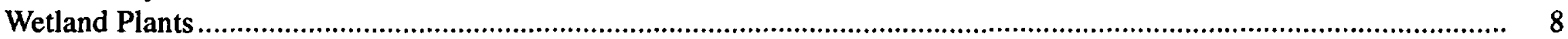

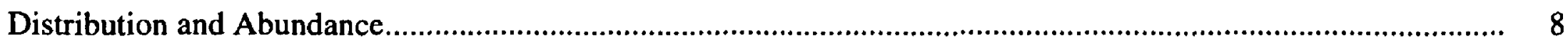

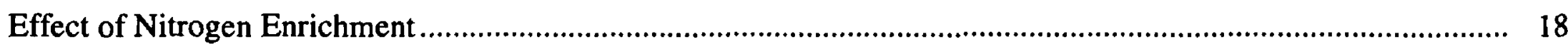

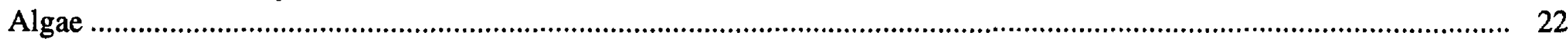



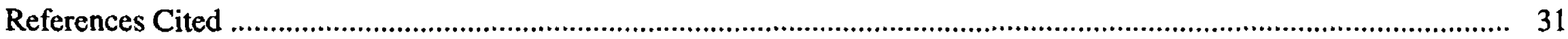

\section{FIGURES}

1-6. Maps showing:

1. Location of Namskaket Marsh and Creek, adjacent septage-treatment facility, and plume of septage-effluent-contaminated ground water, Orleans, Cape Cod, Massachusetts

2. Locations of vegetation and pore-water sampling sites along transects used to measure relative percentage of cover, stem density, and aboveground biomass, Inner Namskaket Marsh, Cape Cod, Massachusetts.

3. Locations of sampling sites used to measure chlorophyll-a concentrations in creek-bottom sediments and leaf-nitrogen content in wetland plants and nitrogen-enrichment experiment sites, Inner Namskaket Marsh

4. Location of experimental (fertilized) and control plots for the nitrogen-enrichment experiment in Spartina and Phragmites vegetation stands, Inner Namskaket Marsh

5. Areal distribution of wetland plants, Inner Namskaket Marsh and Hurley's Bog, August through October, 1995.

6. Pore-water salinity in peat sediment, Inner Namskaket Marsh, July 1995 .............................................. 10

7. Graphs showing aboveground biomass, stem density, and leaf-nitrogen content in experimental (fertilized) and control plots for Phragmites and Spartina, Inner Namskaket Marsh, September 1995 and 1996.

8. Photographs showing distribution of Spartina alterniflora and Spartina patens in nitrogen-enrichment experimental ( $C$ and $D)$ and control ( $A$ and $B$ ) plots, Inner Namskaket Marsh, September 1995

9. Map showing areal distribution of macroalgae in creek channels of upper Namskaket Creek, Inner Namskaket Marsh, July 1994

10. Graphs showing concentrations of chlorophyll- $a$ in creek-bottom sediments, Inner Namskaket Marsh, July 1995 and April and May 1996 and April through October 1996 
TABLES

1. Abundance (relative cover) of wetland plants at sampling sites along transects,

Inner Namskaket Marsh, Cape Cod, Massachusetts, August and September 1995

2. Measured areal coverage of wetland plants and plant associations, Inner Namskaket Marsh,

Cape Cod, Massachusetts, August through October, 1995

3. Wetland plant stem density and aboveground biomass at selected sampling sites,

Inner Namskaket Marsh, Cape Cod, Massachusetts, August and September 1995

4. Wetland plant stem density and leaf-nitrogen content at selected sampling sites,

Inner Namskaket Marsh, Cape Cod, Massachusetts, August 7, 1996

5. Stem density,.aboveground biomass, and leaf-nitrogen content by species in

experimental (fertilized) and control plots of Phragmites australis and

Spartina patens/Spartina alterniflora, Inner Namskaket Marsh,

Cape Cod, Massachusetts, September 1995 and September 1996

6. Chlorophyll- $a$ concentrations in creek-bottom sediments, Inner Namskaket Marsh,

Cape Cod, Massachusetts, April through October, 1996

7. Locations of vegetation and pore-water sampling sites along transects used

to measure relative percentage of cover, stem density, and aboveground

biomass, Inner Namskaket Marsh, Cape Cod, Massachusetts

\section{CONVERSION FACTORS, VERTICAL DATUM, AND WATER-QUALITY UNITS}

CONVERSION FACTORS

\begin{tabular}{|c|c|c|}
\hline Multiply & By & To obtain \\
\hline centimeter $(\mathrm{cm})$ & 0.3937 & inch \\
\hline cubic centimeter $\left(\mathrm{cm}^{3}\right)$ & 0.06102 & cubic inch \\
\hline $\operatorname{gram}(\mathrm{g})$ & 0.03527 & ounce, avoirdupois \\
\hline gram per square meter $\left(\mathrm{g} / \mathrm{m}^{2}\right)$ & 0.0929 & gram per square foot \\
\hline hectare (ha) & 0.003861 & square mile \\
\hline kilometer $(\mathrm{km})$ & 0.6214 & mile \\
\hline liter (L) & 0.2642 & gallon \\
\hline meter (m) & 3.281 & foot \\
\hline meter per day $(\mathrm{m} / \mathrm{d})$ & 3.281 & foot per day \\
\hline square centimeter $\left(\mathrm{cm}^{2}\right)$ & 0.1550 & square inch \\
\hline \multicolumn{3}{|l|}{ microgram per square centimeter } \\
\hline$\left(\mu \mathrm{g} / \mathrm{cm}^{2}\right)$ & 6.45 & microgram per square inch \\
\hline square meter $\left(\mathrm{m}^{2}\right)$ & 10.76 & square foot \\
\hline \multicolumn{3}{|c|}{ 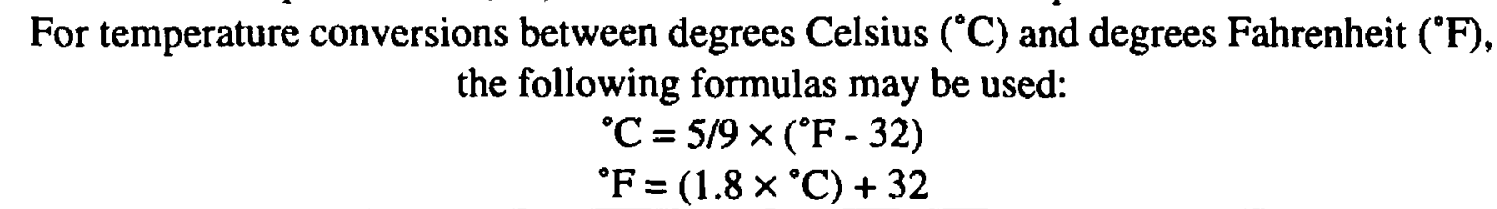 } \\
\hline
\end{tabular}

VERTICAL DATUM

Sea level: In this report, "sea level" refers to the National Geodetic Vertical Datum of 1929 (NGVD of 1929) - a geodetic datum derived from a general adjustment of the first-order level nets of the United States and Canada, formerly called Sea Level Datum of 1929.

\section{WATER-QUALITY UNITS}

Concentrations of chemical constituents are given in milligrams per liter (mg/L). Milligrams per liter is a unit expressing the concentration of chemical constituents in solution as weight (milligrams) of solute per unit volume (liter) of water. For concentrations less than $7,000 \mathrm{mg} / \mathrm{L}$, milligrams per liter is equivalent to "parts per million." 


\title{
Wetland Plants and Algae in a Coastal Marsh, Orleans, Cape Cod, Massachusetts
}

\author{
By Leslie A. DeSimone, Brian L. Howes, Dale G. Goehringer, and Peter K. Weiskel
}

\section{Abstract}

Wetland plants and macro- and microepibenthic algae in inland areas of Namskaket Marsh, Cape Cod, Massachusetts, were surveyed to determine the current (1994-96) distribution and abundance of plant and algal communities. The study area consists of a 7-hectare inland lobe of Namskaket Marsh (Inner Namskaket Marsh) and an adjacent 1.3-hectare brackish marsh, Hurley's Bog. Discharge of a plume of effluent-contaminated ground water that originates at a nearby septage-treatment facility could potentially increase nitrogen loading to both of these wetland areas. A small-scale nitrogen-enrichment experiment also was conducted in the Inner Namskaket Marsh during 1995-96 in order to determine the potential effects of increased nitrogen enrichment on two dominant wetland plant associations.

Inner Namskaket Marsh is a typical New England high salt marsh with brackish-water transition zones along the marsh/upland boundary. Associations of Phragmites australis (common reed), Spartina patens (salt-marsh hay), Spartina alterniflora (cordgrass), and Distichlis spicata (spike grass) cover about three-fourths of the site and broadly reflect the distribution of tidal flooding and ground-water discharge. Other commonly occurring plants are Iva frutescens (marsh elder), Spartina cynosuroides (big cordgrass), Typha sp. (cattail), Scirpus robustus (robust bulrush), Salicornia europaea, (common glasswort), Pluchea purpurascens (salt-marsh fleabane), and Solidago sempervirens (seaside goldenrod). Wrack deposits (dead plant material) also covered almost 15 percent of the mapped area in 1995 and appeared to be a significant factor affecting plant distribution.

Hurley's Bog is a brackish marsh, connected to Inner Namskaket Marsh through a culvert in a railroad embankment, that is reverting to a freshwater wetland. The bog consists of an open, herbaceous area surrounded by shrub oak/maple wet woodland. The open area is dominated by Phragmites australis (about 80 percent coverage), with Iva frutescens, Scirpus robustus, Solidago sempervirens, and Spartina patens present to a lesser degree.

Several quantitative characteristics of the marsh plant community that could change with increased nitrogen loading were measured. Aboveground biomass, an indicator of annual production, ranged from 390 to 1,050 grams of dry weight per square meter for various mixed associations of primarily Spartina patens, Spartina alterniflora, and Distichlis spicata. Leaf-nitrogen content, which can be a measure of nitrogen fertilization, was determined for Phragmites australis (3.18 percent of dry weight) and Scirpus robustus (1.87 percent of dry weight) in areas along the marsh/upland border where ground-water seepage is greatest.

The results of a small-scale nitrogen-enrichment experiment indicated that nitrogen addition affected plant growth in Phragmites australis and Spartina patens/Spartina alterniflora plots. Aboveground biomass and leaf-nitrogen content of Phragmites increased under nitrogen addition. In the $S$. patens/ $S$. alterniflora associations, nitrogen addition resulted in a species shift from $S$. patens to $S$. alterniflora rather than in an increase in aboveground biomass during the two growing seasons of the experiment.

Macro- and micro-epibenthic algae were surveyed in the tidal creeks of Inner Namskaket Marsh, which are the areas most likely to experience a change in nitrogen loading from nitrogen-contaminated ground water. Macroalgae, surveyed in July and August 1994, consisted mostly of Rhizoclonium, a very fine, green filamentous alga, and Bangia, a filamentous red alga. Microalgae formed dense populations in some areas and were surveyed by measuring the distribution of chlorophyll- $a$ concentrations in sediments. Marshwide surveys showed that chlorophyll- $a$ concentrations were highly variable at several scales of measurement and with time and ranged from 0.2 to 95 -micrograms per square centimeter in April and May 1996. 


\section{INTRODUCTION}

Namskaket Marsh is an 80-hectare salt marsh on the northeastern coast of Cape Cod, Massachusetts (fig. 1). It occupies a triangular indentation in the coast sheltered by barrier dunes and beach sands (Oldale and others, 1971) and is underlain by fibrous peat, as is typical of marshes along the northern Atlantic coast of the United States (Niering and Warren, 1980). Like other mature New England salt marshes, Namskaket Marsh supports a flowering plant community of Spartina alterniflora (cordgrass), Spartina patens (saltmarsh hay), Distichlis spicata (spike grass), and other wetland angiosperms, epibenthic algae, and abundant populations of invertebrates, fish, and birds (Nixon, 1982; Frey and Basan, 1985; Teal, 1986). In addition to providing wildlife nurseries and habitat, salt marshes such as Namskaket form storm buffers, filter many waterborne contaminants, and are sites of groundwater discharge (Valiela and Teal, 1979; Mitsch and Gosselink, 1993). In recognition of its natural beauty and critical value to environmental quality, Namskaket Marsh has been designated as an Area of Critical Environmental Concern and an Outstanding Resource Water by the State of Massachusetts [Massachusetts General Law, chapter 21a, sections 2(7) and 40(e)].

Inland parts of Namskaket Marsh are areas of ground-water discharge. A plume of nitrogen-rich ground water is present in the aquifer near the marsh and is moving with regional flow toward discharge areas in the marsh and Cape Cod Bay. The nitrogenrich plume originates from an adjacent septagetreatment facility (fig. 1). The facility, which began operating in 1990 , discharges effluent containing 40 to $50 \mathrm{mg} / \mathrm{L}$ of total nitrogen to the highly permeable glacial aquifer (DeSimone and Howes, 1995; DeSimone and others, 1996; J. Burgess, Orleans, Brewster, and Eastham Ground-Water Protection District, written commun., 1990-97). The resulting plume of effluent-contaminated ground water has traveled at about $0.1 \mathrm{~m} / \mathrm{d}$ toward the marsh since its inception. Nitrogen within the plume has undergone little attenuation during ground-water transport and is still largely mobile (DeSimone and Howes, 1996; Weiskel and others, 1996, 1997). Nitrogen concentrations directly affect the productivity and species composition of salt-marsh plants and algae and indirectly affect the ecology of herbivores and detritivores (Valiela and others, 1985; Teal, 1986; Mitsch and Gosselink, 1993). Thus, increased nitrogen loading to Namskaket Marsh from discharge of the effluent-contaminated ground water is of concern because it may alter the distribution and(or) productivity of wetland plants and algae and indirectly affect other wildlife in the marsh. Increased nitrogen loading also is a potential consequence of increased residential and commercial development throughout the ground-water contributing area and drainage basin of the marsh (Giblin and Gaines, 1990; Valiela and others, 1990; Weiskel and Howes, 1992).

The U.S. Geological Survey (USGS), in cooperation with the Massachusetts Department of Environmental Protection, Division of Watershed Management (MDEP), has monitored the movement of effluent-contaminated ground water through the aquifer since the beginning of effluent discharge. In addition, the USGS has been investigating hydrologic, water-quality, biogeochemical, and ecological conditions in Inner Namskaket Marsh since 1993 in collaboration with the Woods Hole Oceanographic Institution and in cooperation with the MDEP and Cape Cod Commission.

This report presents a baseline spatial distribution and abundance of wetland plants and macro- and micro-epibenthic algae in a 7-hectare area of Inner Namskaket Marsh and in adjacent Hurley's Bog. Hurley's Bog is a 1.3-hectare brackish marsh that is connected with Namskaket Marsh through a culvert in a railroad embankment (now converted to a bike trail), which restricts tidal flow (fig. 1). These areas are immediately downgradient from the treatment facility. The spatial distribution and abundance of wetland plants and algae in the marsh and bog were determined by direct measurement and interpretation of aerial photographs during 1994-96. In addition to the spatial data, quantitative data on stem density, aboveground biomass, and leaf-nitrogen content of wetland plants and chlorophyll- $a$ concentrations in creek sediments were collected because these characteristics are sensitive to changes in nitrogen loading to the marsh. A small-scale nitrogen-enrichment experiment also was conducted in vegetation stands of $S$. patens/ S. alterniflora and Phragmites australis in the inner marsh during 1995-96 in order to determine the potential effects of increased nitrogen enrichment on these two dominant wetland plant associations. 


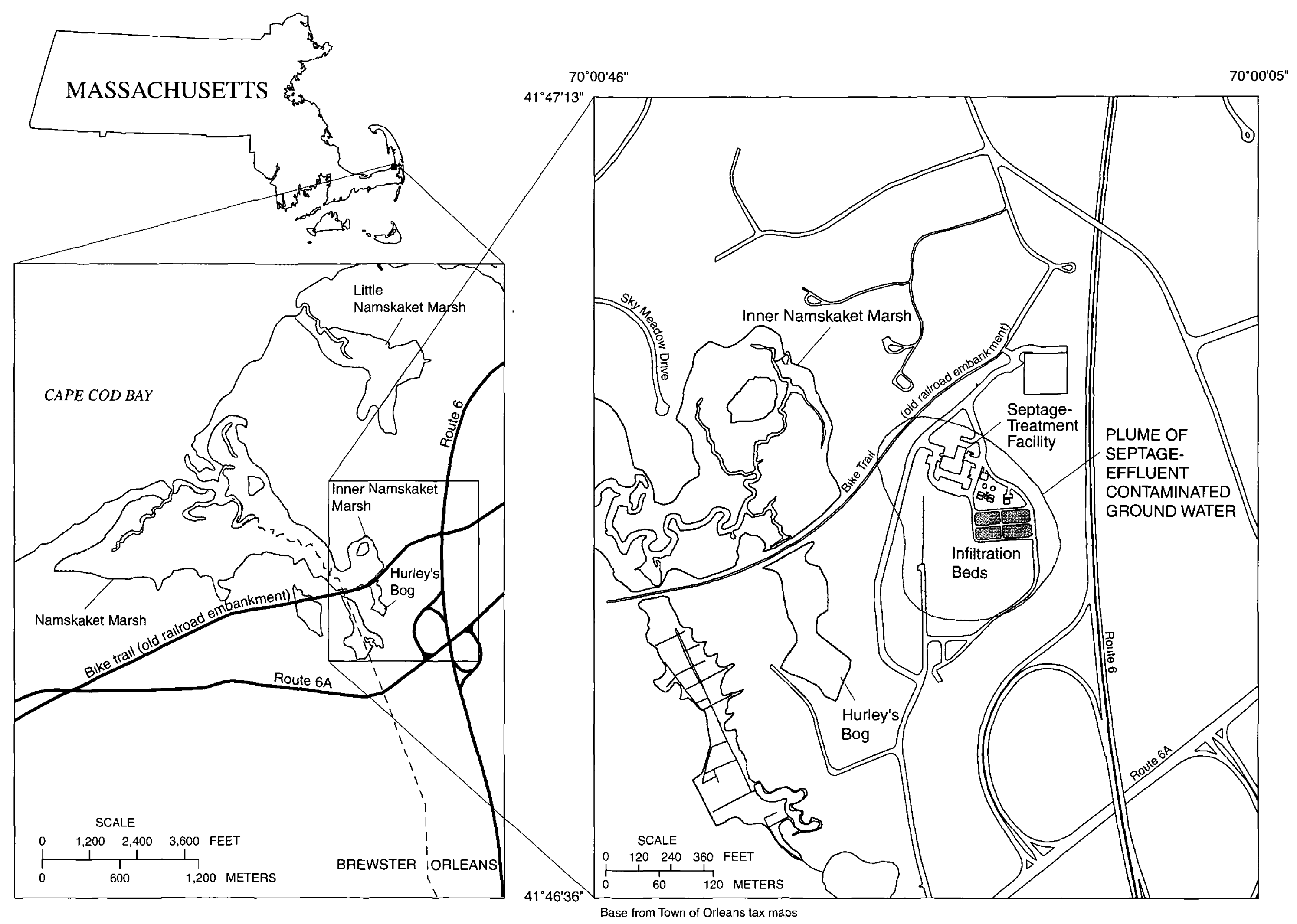




\section{STUDY METHODS}

\section{Data Collection}

Spatial data on the distribution and abundance of wetland plants in inner Namskaket Marsh were collected using a network of 114 sampling sites arranged in 16 transects (fig. 2). The transects were laid out in English units of measurement and spaced at about 150-foot (45-m) intervals and oriented perpendicular to the marsh/upland boundary; sampling sites were located along each transect at 70- to 75-foot (21- to 22-m) intervals (more densely spaced near the marsh/upland boundary) except where creeks made regular spacing impossible. Pore-water sampling sites were marked with $2-\mathrm{cm}$ diameter, 40 to $50-\mathrm{cm}$ long

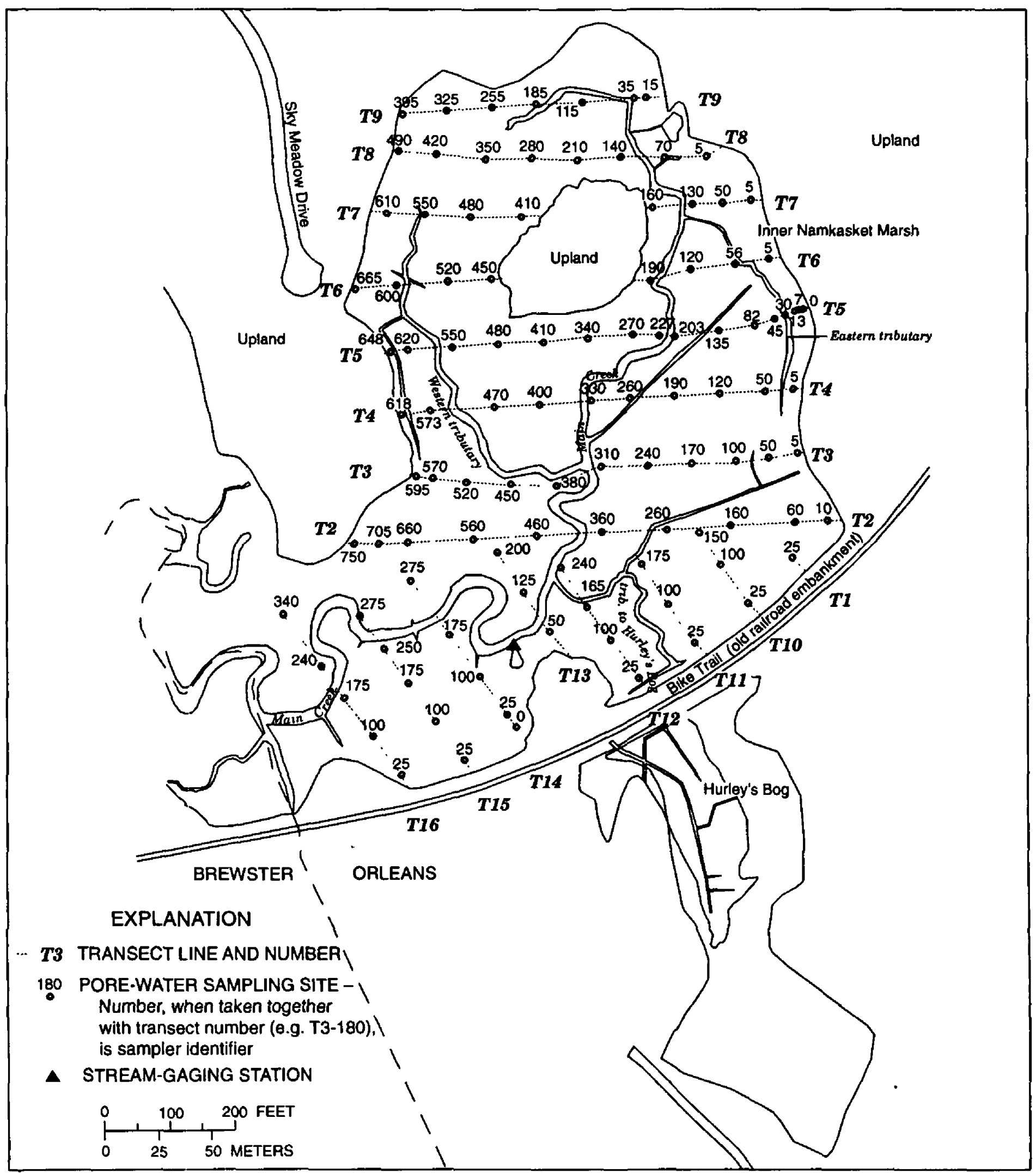

Figure 2. Locations of vegetation and pore-water sampling sites along transects used to measure relative percentage of cover, stem density, and aboveground biomass, Inner Namskaket Marsh, Cape Cod, Massachusetts. Sampling-site identifier indicates transect number and distance, in feet, from the marsh/upland boundary. 
polyvinylchloride pipes, driven 10 to $15 \mathrm{~cm}$ into the marsh sediment; the pipes were slotted at the bottom to allow for collection of pore-water samples for salinity determination. Samplers were located in state-plane coordinates relative to established benchmarks (J. Eyestone, Schofield Brothers, Inc., written commun., 1990) using a Nikon Total Station (table 7, at back of report).

Abundance of plant species was estimated at most sampling sites by visual estimation of relative percentage of cover of each species within $1 \mathrm{~m}$ of the sampler. Fifteen sampling sites along transects 2, 3, 7, and 13-16 (fig. 2) were chosen as representative plant stands based on this marshwide survey for measurement of distribution, stem density, and biomass per unit area. These characteristics were measured by harvesting all stems within duplicate $0.1-\mathrm{m}^{2}$ circular quadrats, randomly placed near each sampling site. Samples for determination of baseline leaf-tissue nitrogen content were collected from monospecific stands of the dominant plant species at 15 sampling sites along the marsh/upland border (fig. 3). The top three fully unfurled leaves from each of six plants within a $0.5-\mathrm{m}^{2}$ quadrat were removed at the leaf base; the 18 leaves were grouped into two replicate subsamples (three plants or nine leaves per replicate). Stem density in the quadrat and the height of the harvested plants also were measured.

A vegetation map of Inner Namskaket Marsh was prepared at a scale of 1:1,000 on a base showing the marsh/upland boundary, creeks, and sampling sites. The relative percentage of cover, stem density, and aboveground biomass data at the regularly spaced sampling sites were used to identify pure stands and the dominant mixed-assemblage associations of plants in the marsh at these locations. The spatial extent of the plant associations was estimated from color aerial photographs of the marsh (scales approximately 1:600 to 1:1,200, October 12,1995, Kelsey-Kennard Photographers, Chatham, Mass.); the locations of selected sampling sites were marked in the field with brightly colored plastic sheets for identification in the photographs. Boundaries between plant associations were hand-drawn on the base map, using the sampling sites for spatial reference. Vegetation patches smaller than about $4 \mathrm{~m}^{2}$ were not mapped separately. The hand-drawn map was scanned and screen-digitized into a Geographic Information System data layer. The distribution of wetland plants in Hurley's Bog was mapped with a measuring tape with reference to creek and bog-boundary landmarks (the creek center, prominent bends in the creek and junctions with tributaries, the culvert, the base of the embankment, and the edge of trees) that were located with the Nikon Total Station.

Chlorophyll- $a$ concentrations in creek-bottom sediments were measured to assess the distribution and biomass of epibenthic microalgae in the creek; macroalgal occurrence was determined by direct field observation and taxonomic identification of harvested samples. Sediment samples for analysis of chlorophyll- $a$ concentrations were collected repeatedly from as many as 25 sampling sites in 1995 and 1996 (fig. 3). Sampling sites in 1996 were located at approximately regular intervals ( 30 to $45 \mathrm{~m}$ ) along the main creek, in most cases near transects. Along the eastern tributary and upper part of the main creek, sites were more densely spaced because ground-water discharge is concentrated in those areas (10- to $25-\mathrm{m}$ intervals; site 23 was added to coincide with a creekwater sampling site). Along the western tributary, site selection was limited by the large amount of wrack covering the creek bottom. In 1995, sampling sites were along the eastern tributary and main creek only and were more densely spaced (5-m intervals). At each site, $15 \mathrm{~cm}^{3}$ of sediment was collected with a small piston corer $\left(5 \mathrm{~cm}^{2}\right.$ by $\left.3 \mathrm{~cm}\right)$ from midway between the center and bank edge of the tidal creek (1995, one sample per site) or from the center and both margins of the submerged creek bottom at low tide (1996; three samples per site). Samples were kept in sealed test tubes at $4^{\circ} \mathrm{C}$ in the dark after collection for transport to the laboratory.

\section{Nitrogen-Enrichment Experiment}

A nitrogen-enrichment experiment was conducted in stands of $S$. patens/S. alterniflora and Phragmites australis. Duplicate 2- $\mathrm{m}^{2}$ ( $S$. patens/ S. alterniflora) and $4-\mathrm{m}^{2}$ (Phragmites) plots (figs. 3 and 4) received weekly nitrogen applications during the growing seasons (May through August) of 1995 and 1996. The fertilization rate of $0.725 \mathrm{~g} /\left(\mathrm{m}^{2} / \mathrm{d}\right)$ of $\mathrm{N}$ represents the maximum estimated loading rate associated with discharge of the existing plume of effluent-contaminated ground water. Nitrogen was applied as sodium nitrate dissolved in 8 to $10 \mathrm{~L}$ of creek water. Duplicate control plots in each stand received equal volumes of unamended creek water. Duplicate 


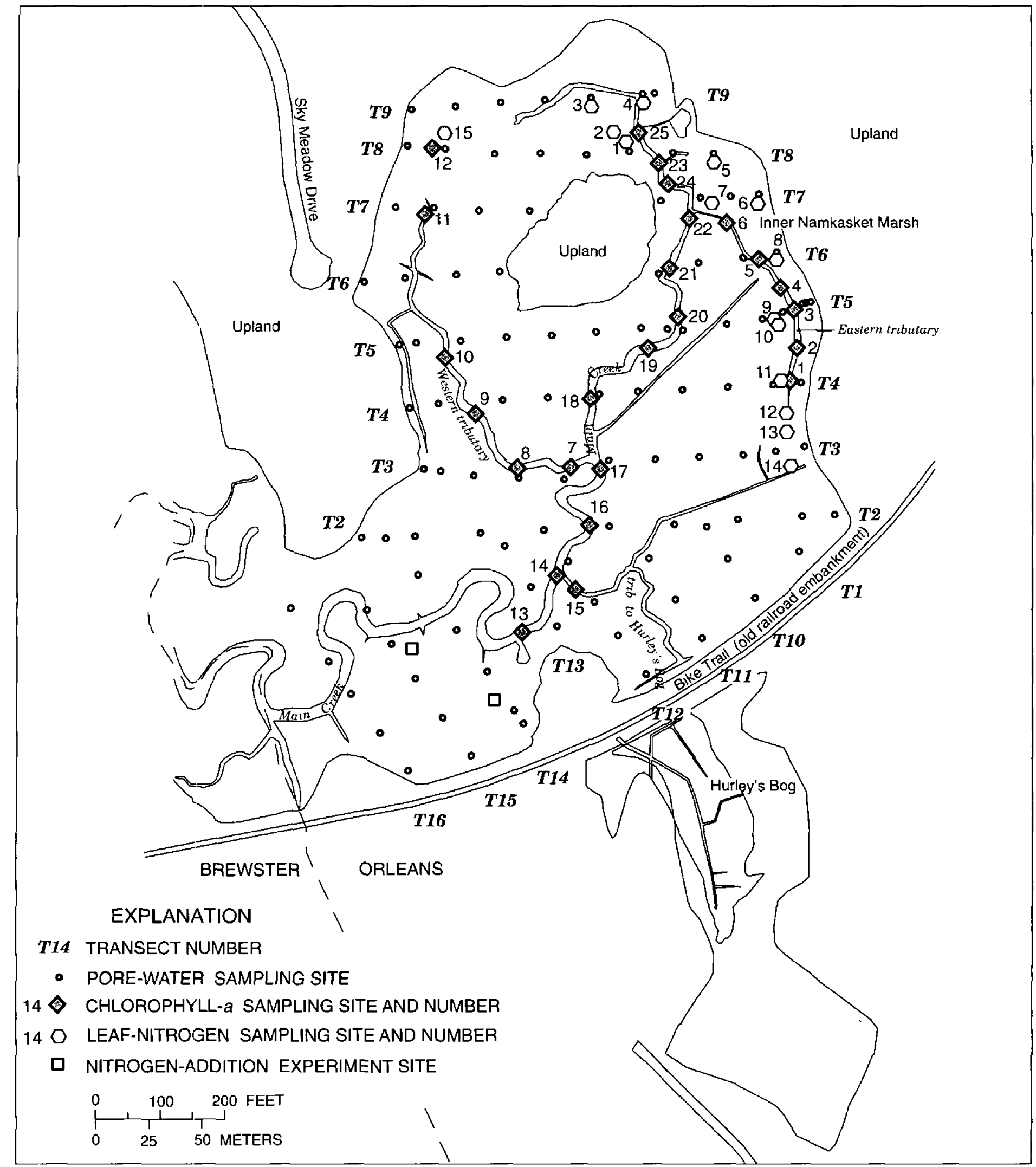

Figure 3. Locations of sampling sites used to measure chlorophyll-a concentrations in creek-bottom sediments and leaf-nitrogen content in wetland plants and nitrogen-enrichment experiment sites, Inner Namskaket Marsh, Cape Cod, Massachusetts.

subplots of experimental and control plots were harvested at the end of the growing season on September 11, 1995, and September 12, 1996, by removing all stems within $0.1-\mathrm{m}^{2}$ circular ( $S$. patens/ S. alterniflora) and $0.5-\mathrm{m}^{2}$ square (Phragmites) quadrats.

\section{Analytical Methods}

Harvested plant samples were sorted by species for counts of stem density and weight of aboveground biomass after separation into live and dead stems. Live and dead stems were dried at $60^{\circ} \mathrm{C}$ for 72 hours and 

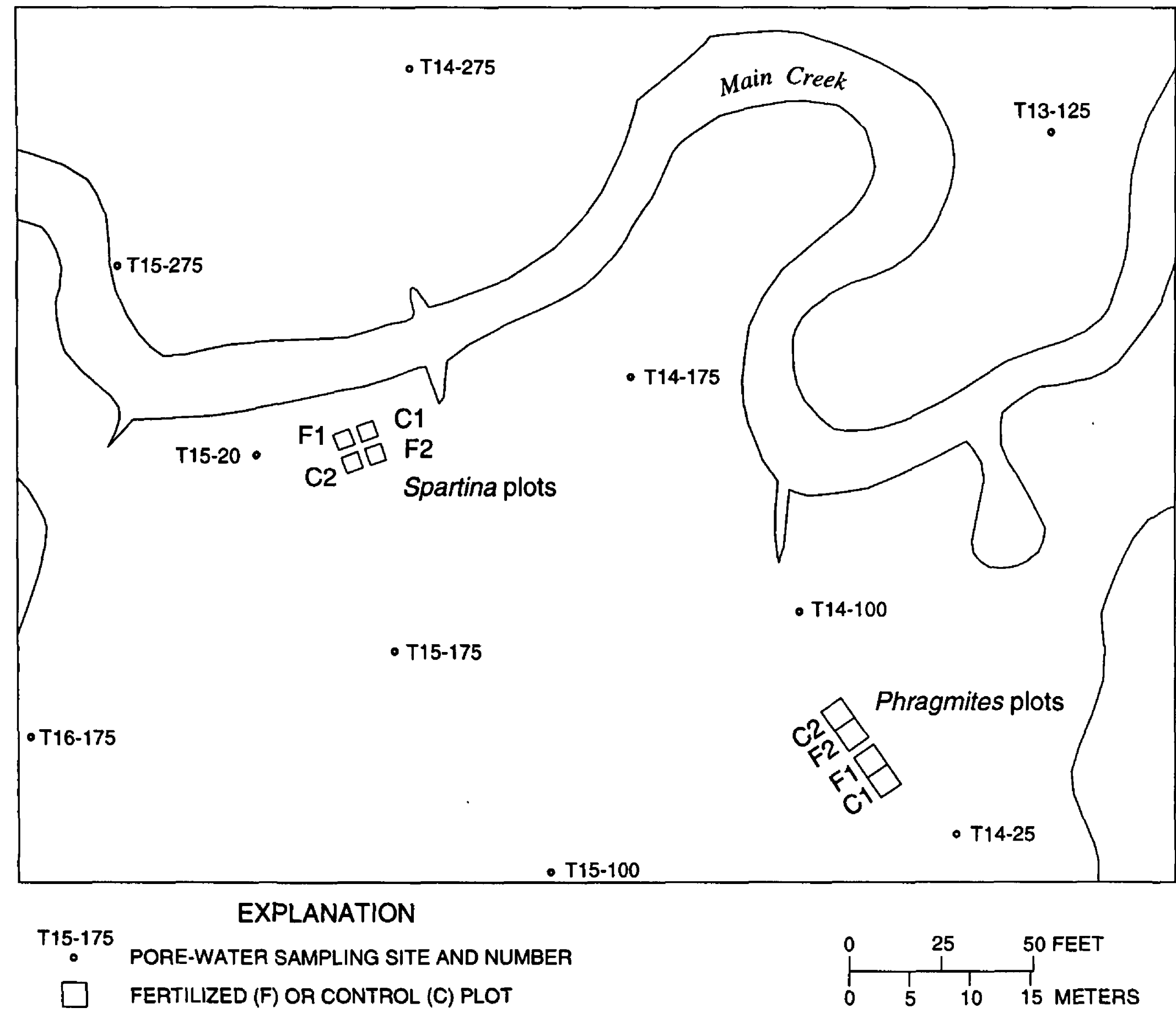

Figure 4. Location of experimental (fertilized) and control plots for the nitrogen-enrichment experiment in Spartina and Phragmites vegetation stands, Inner Namskaket Marsh, Cape Cod, Massachusetts.

weighed separately (aboveground biomass). The 10 tallest stems in each sample from the experimental plots were determined as an indirect measure of productivity for possible future monitoring efforts by measuring the 12 tallest stems (determined visually) and discarding the two lowest measurements. Leaftissue nitrogen content was measured on dried, ground samples with a CHN elemental analyzer (Perkin-Elmer PE2400); the accuracy of the analysis was \pm 5 percent and was maintained by including a blank or standard after every seven samples. Creek-bottom sediment samples for analysis of chlorophyll- $a$ concentrations were extracted with an acetone solution $(1 \mathrm{~g}$ of magnesium carbonate per liter of 100 percent acetone, $30 \mathrm{~cm}^{3}$ ), which was added in the laboratory within 2 to 6 hours of sample collection. Samples were extracted for several days at $-20^{\circ} \mathrm{C}$ before centrifugation and spectrophotometric analysis (Bausch and Lomb Spectronic 2000). Chlorophyll- $a$ was determined from absorbance before and after acidification with hydrochloric acid (Parsons and others, 1989). 


\section{WETLAND PLANTS}

\section{Distribution and Abundance}

The large-scale distribution of wetland plants in a salt marsh is controlled primarily by physical and chemical conditions related to the frequency and duration of tidal flooding (Mitsch and Gosselink, 1993). The frequency of tidal flooding is determined by topography and defines "low" and "high" marsh areas. Low marsh areas are flooded by most high tides and are dominated by monospecific stands of Spartina alterniflora in New England marshes (Teal, 1986; Tiner, 1987). High marsh areas support more diverse vegetation and are flooded less frequently, generally by the bimonthly spring tides and storm-surge tides (Nixon, 1982). In terms of flooding frequency and saltmarsh vegetation, Inner Namskaket Marsh is mostly high marsh with a brackish-water transition zone along the marsh/upland border; $S$. alterniflora is primarily restricted to creek banks. The median salinity of creek waters at high tide measured in the main channel to the inner marsh (gaging station, fig. 2) is $17 \mathrm{ppt}$, which is significantly less than the salinity of the source waters in Cape Cod Bay (30 ppt). The salinity of the creek waters is significantly less than seawater salinity because of ground-water discharge through the tidalcreek sediments and at the marsh/upland boundary (Howes and others, 1996).

The relative importance of plant species in a wetland can be evaluated in terms of the area colonized by and the density of the species in pure stands and in mixed-species assemblages and their contribution (relative percentage of cover) in mixed assemblages. The dominant wetland plants in Inner Namskaket Marsh were defined from the frequency of occurrence and relative percentage of cover at each of the sampling sites (table 1) and measured areal coverage (table 2), shown in figure 5. The predominant rooted macrophytes were Phragmites australis (common reed), Spartina patens, Distichlis spicata, and Spartina alterniflora, which occurred at 46, 22, and 18 percent of the sampled sites, respectively (table 1). Pure stands and mixed assemblages of these four species covered 73 percent of the mapped vegetated area (table 2). Other commonly occurring species were Iva frutescens (marsh elder shrub), Spartina cynosuroides (big cordgrass), Scirpus robustus (robust bulrush), Solidago sempervirens (seaside goldenrod), Pluchea purpurascens (salt-marsh fleabane), Salicornia europaea (common glasswort), and Typha sp. (cattail); these species generally occurred at less than 10 percent of the sampled sites and were not spatially dominant (tables 1 and 2), Juncus gerardi (black grass), commonly a major component of high marsh vegetation in New England along upland borders (Redfield, 1972; Niering and Warren, 1980; Nixon, 1982), was much less common than other brackish wetland plants in Inner Namskaket Marsh.

The major plant associations in Inner Namskaket Marsh form a mosaic (fig. 5). Spatial distribution of the dominant species, Phragmites australis, $S$. patens, $S$. alterniflora, and Distichlis spicata, broadly reflected the distribution of tidal flooding and ground-water discharge. As is typical of salt marshes along the Atlantic Coast, the creek banks were colonized by S. alterniflora (tall form) except in the innermost brackish areas. S. patens and Distichlis spicata occurred in patches primarily inland from creek banks. Phragmites australis, a fresh- to brackish-water plant, occurred primarily in irregular bands along the marsh/ upland boundary, where pore-water salinities of 4 to 8 ppt and less (fig. 6) reflect the nearly continuous ground-water seepage zones in these areas. Phragmites australis near transects T8 and T9 was particularly luxuriant, forming dense, monospecific stands exceeding $3 \mathrm{~m}$ in height (figs. 3 and 5). Other plants tolerant of brackish water, $S$. cynosuroides, Typha sp., Iva frutescens, and Scirpus robustus, also occurred near marsh/upland boundaries. These broad patterns, which are typical of salt marshes in New England, result from a complex interaction of environmental factors (flooding frequency and duration, salinity, nutrient availability, redox conditions, and other factors) and interspecific competition (Miller and Egler, 1950; Nixon, 1982; Dacey and Howes, 1984; Bertness and Ellison, 1987; Bertness, 1992).

In addition to the large-scale distributions, which tend to be relatively stable, smaller-scale patchiness that appears to result from physical disturbance also was apparent in Inner Namskaket Marsh (Hartman, 1988). Large deposits of wrack, consisting mostly of dead $S$. alterniflora stems broken off by floating ice in winter (Bertness, 1992), were common. 


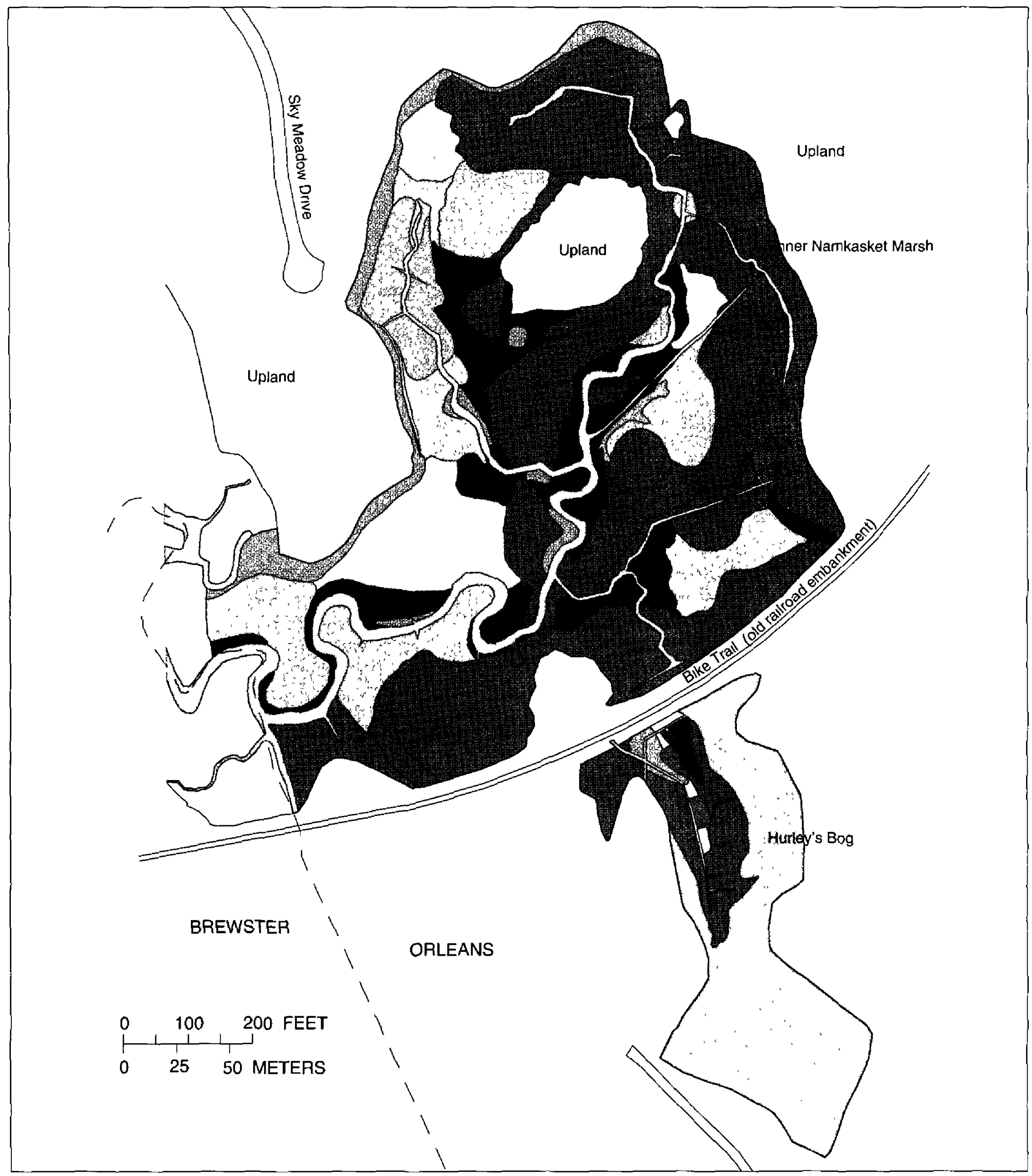

\section{EXPLANATION}

Phragmites australis

Spartma patens

Spartina alterniflora

S. patens / D. specate

Iva frutescens

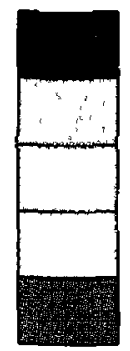

Distichlis spicata

Spartina cynosuroides

S. patens / $S$ cynosurotdes

D. spicata/S. robustus

Typhasp

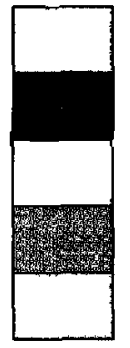

Salicornia $\mathrm{sp}$

Scirpus robustus

Solidago sp. /S. patens

Phragmutes / Solidago sp.

Shrub oak / maple wet woodland

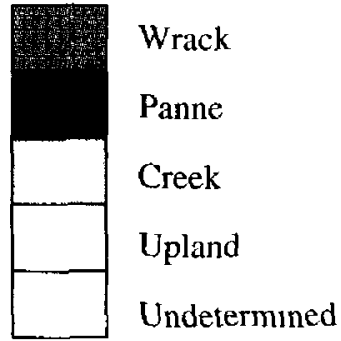

Figure 5. Areal distribution of wetland plants, Inner Namskaket Marsh and Hurley's Bog, Cape Cod,

Massachusetts, August through October, 1995. This map was compiled at 1:1,000 scale from measurements of relative percentage of cover, stem density, and aboveground biomass at sampling sites (see figure 2 and tables 1 and 3) and color aerial photographs (scales of 1:600 to 1:1,200) of the marsh taken by Kelsey-Kennard Photographers, Chatham, Massachusetts on October 12, 1995. 


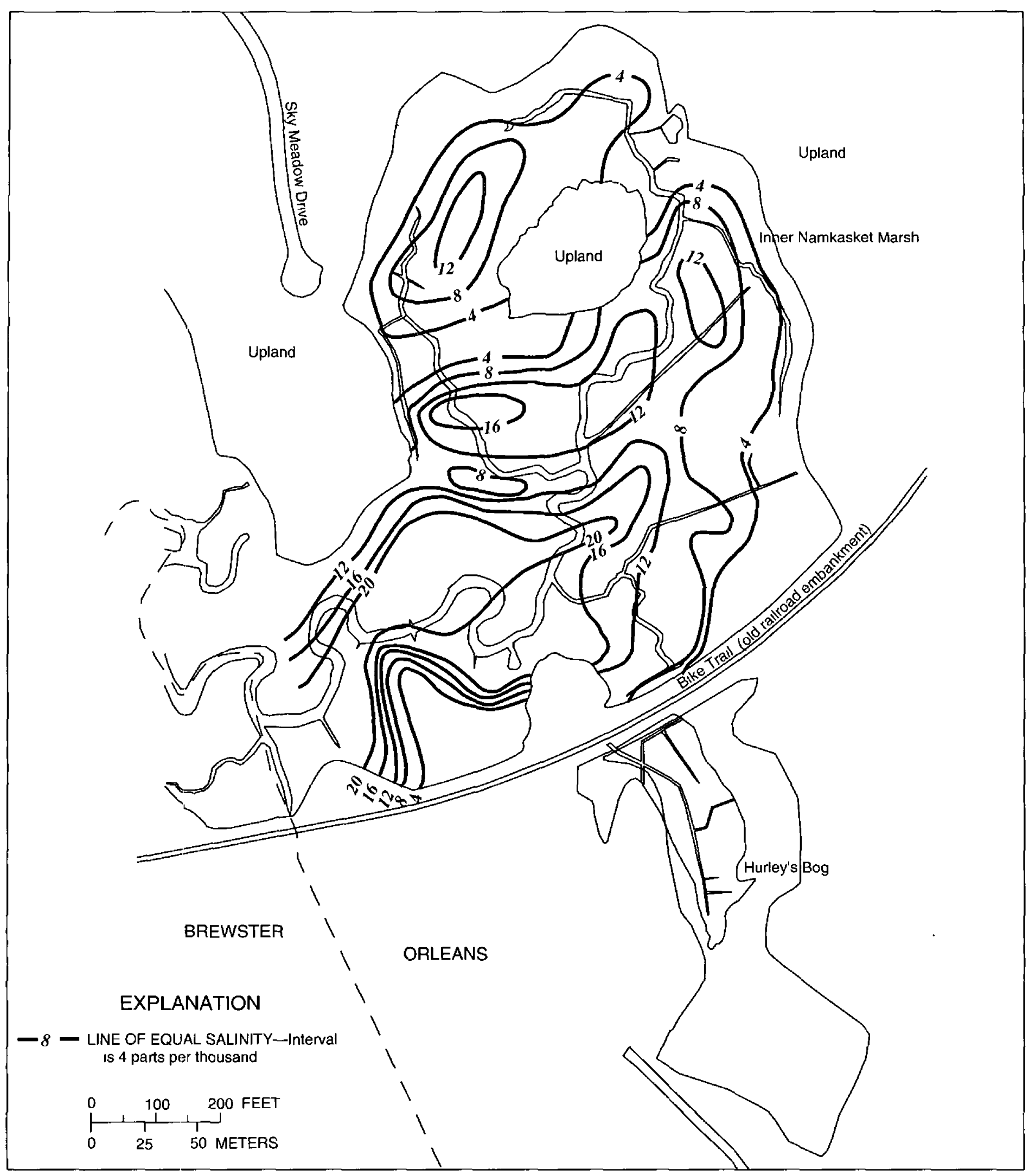

Figure 6. Pore-water salinity in peat sediment, Inner Namskaket Marsh, Cape Cod, Massachusetts, July 1995. 
Table 1. Abundance (relative cover) of wetland plants at sampling sites along transects, Inner Namskaket Marsh, Cape Cod, Massachusetts, August and September 1995

[Salicornia europaea: Includes small amounts of S. bigelovii and S. virginica. Grass: Includes other upland plants as indicated. Abundance determined at sampling site unless otherwise indicated. ND, not determined; < actual value is less than value shown; >, actual value is greater than value shown]

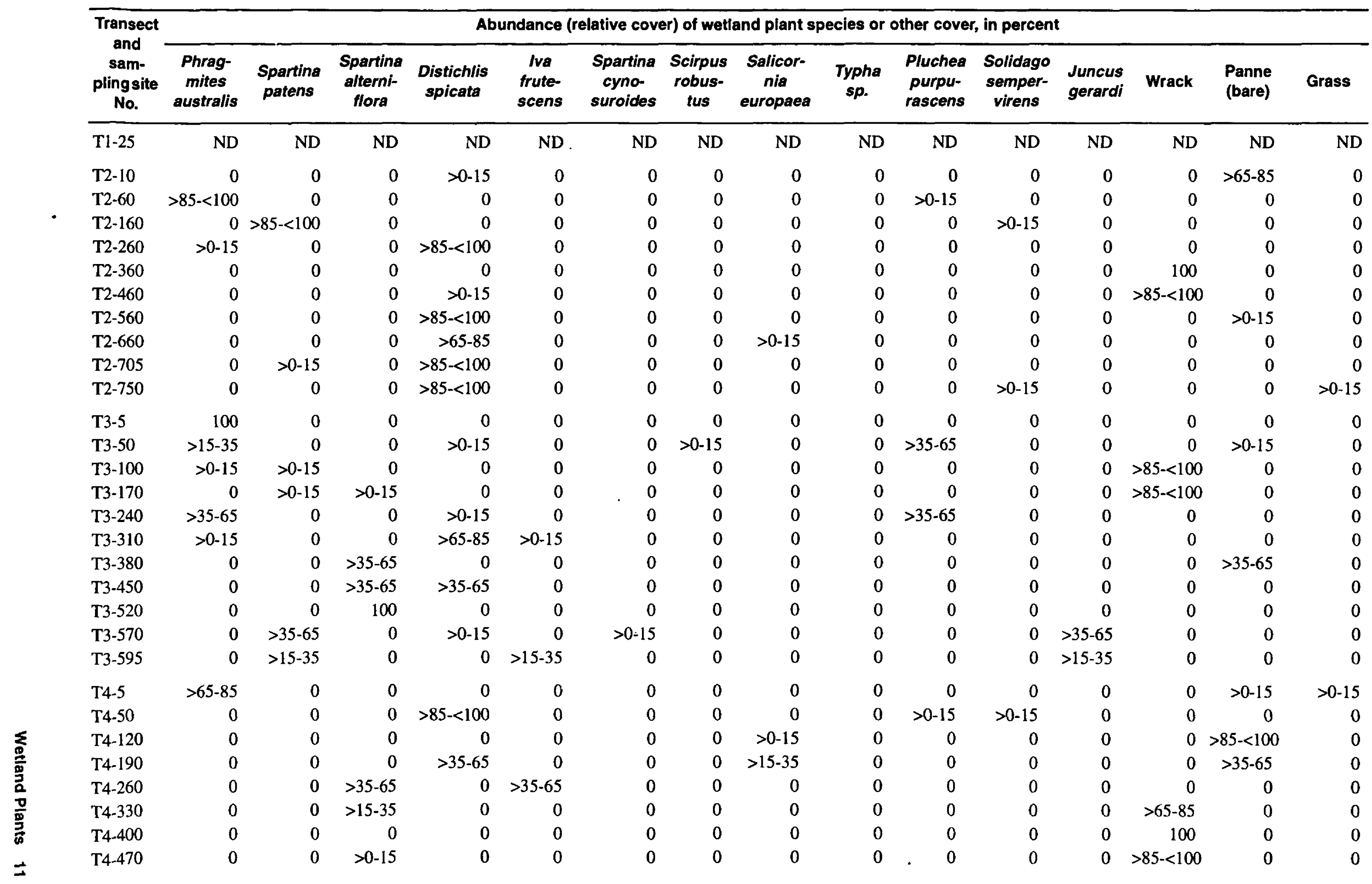




\begin{tabular}{|c|c|c|c|c|c|c|c|c|c|c|c|c|c|c|c|}
\hline \multirow{2}{*}{$\begin{array}{l}\text { Transect } \\
\text { and } \\
\text { sam- } \\
\text { plingsite } \\
\text { No. }\end{array}$} & \multicolumn{15}{|c|}{ Abundance (relative cover) of wetland plant species or other cover, in percent } \\
\hline & $\begin{array}{c}\text { Phrag- } \\
\text { mites } \\
\text { australis }\end{array}$ & $\begin{array}{c}\text { Spartina } \\
\text { patens }\end{array}$ & $\begin{array}{l}\text { Spartina } \\
\text { alterni- } \\
\text { flora }\end{array}$ & $\begin{array}{l}\text { Distichlis } \\
\text { spicata }\end{array}$ & $\begin{array}{c}\text { Iva } \\
\text { frute- } \\
\text { scens }\end{array}$ & $\begin{array}{l}\text { Spartina } \\
\text { cyno- } \\
\text { suroides }\end{array}$ & $\begin{array}{c}\text { Scirpus } \\
\text { robus- } \\
\text { tus }\end{array}$ & $\begin{array}{l}\text { Salicor- } \\
\text { nia } \\
\text { europaea }\end{array}$ & $\begin{array}{c}\text { Typha } \\
\text { sp. }\end{array}$ & $\begin{array}{l}\text { Pluchea } \\
\text { purpu- } \\
\text { rascens }\end{array}$ & $\begin{array}{c}\text { Solidago } \\
\text { semper- } \\
\text { virens }\end{array}$ & $\begin{array}{l}\text { Juncus } \\
\text { gerardi }\end{array}$ & Wrack & $\begin{array}{l}\text { Panne } \\
\text { (bare) }\end{array}$ & Grass \\
\hline T4-573 & $>35-65$ & 0 & $>35-65$ & 0 & 0 & $>0-15$ & 0 & 0 & 0 & $>0-15$ & 0 & 0 & 0 & 0 & $>0-15$ \\
\hline T4-618 & 0 & $>35-65$ & 0 & 0 & 0 & 0 & 0 & 0 & 0 & 0 & $>0-15$ & 0 & 0 & 0 & $>35-65$ \\
\hline T5-0 & ND & ND & ND & ND & ND & $\dot{N D}$ & ND & ND & ND & ND & $N D$ & $N D$ & ND & ND & ND \\
\hline T5.7 & ND & ND & ND & ND & ND & ND & ND & ND & ND & ND & ND & ND & ND & ND & ND \\
\hline T5-13 & $>35-65$ & 0 & 0 & 0 & 0 & 0 & 0 & 0 & 0 & 0 & 0 & 0 & 0 & $>35-65$ & 0 \\
\hline T5-30 & 100 & 0 & 0 & 0 & 0 & 0 & 0 & 0 & 0 & 0 & 0 & 0 & 0 & 0 & 0 \\
\hline T5-45 & 100 & 0 & 0 & 0 & 0 & 0 & 0 & 0 & 0 & 0 & 0 & 0 & 0 & 0 & 0 \\
\hline T5-82 & 0 & 0 & 0 & 0 & 0 & 0 & 0 & 0 & 0 & 0 & 0 & 0 & 100 & 0 & 0 \\
\hline T5-135 & 0 & 0 & 0 & 0 & 0 & 0 & 0 & 0 & 0 & 0 & 0 & 0 & 100 & 0 & 0 \\
\hline T5-203 & $>35-65$ & 0 & 0 & $>35-65$ & $>0-15$ & 0 & 0 & 0 & 0 & 0 & $>0-15$ & 0 & 0 & 0 & 0 \\
\hline T5-227 & 0 & 0 & 0 & 0 & 0 & $>85-<100$ & 0 & 0 & 0 & 0 & $>0-15$ & 0 & 0 & 0 & 0 \\
\hline T5-340 & 0 & 0 & 0 & 0 & 0 & 0 & 0 & 0 & 0 & 0 & 0 & 0 & 100 & 0 & 0 \\
\hline T5-410 & $>15-35$ & $>0-15$ & 0 & $>15-35$ & 0 & 0 & 0 & 0 & 0 & 0 & $>15-35$ & 0 & 0 & $>0-15$ & 0 \\
\hline T5-480 & $>0-15$ & 0 & 0 & $>65-85$ & 0 & 0 & 0 & 0 & 0 & 0 & $>0-15$ & 0 & 0 & 0 & 0 \\
\hline T5.550 & 0 & 0 & 0 & $>35-65$ & 0 & $>15-35$ & 0 & 0 & 0 & 0 & $>15-35$ & 0 & 0 & 0 & 0 \\
\hline T5-620 & 0 & 0 & 0 & 0 & $>0-15$ & $>65-85$ & 0 & 0 & 0 & 0 & $>15-35$ & 0 & 0 & 0 & 0 \\
\hline T5-648 & 0 & 0 & 0 & 0 & 0 & 0 & 0 & 0 & 0 & 0 & $>0-15$ & 0 & 0 & 0 & $>85-<100$ \\
\hline T6-5 & $>85-<100$ & 0 & 0 & 0 & 0 & 0 & 0 & 0 & 0 & 0 & 0 & 0 & 0 & $>0-15$ & 0 \\
\hline T6-56 & ND & ND & ND & ND & ND & ND & ND & ND & ND & ND & ND & ND & ND & ND & ND \\
\hline T6- 120 & $>35-65$ & 0 & 0 & $>15-35$ & $>35-65$ & 0 & 0 & 0 & 0 & 0 & 0 & 0 & 0 & 0 & 0 \\
\hline T6-190 & ND & ND & ND & ND & ND & ND & ND & ND & ND & ND & ND & ND & ND & ND & ND \\
\hline T6-450 & 0 & 0 & 0 & $>65-85$ & 0 & 0 & 0 & 0 & 0 & 0 & $>15-35$ & 0 & 0 & 0 & 0 \\
\hline T6-520 & 0 & 0 & 0 & $>65-85$ & 0 & 0 & 0 & 0 & 0 & 0 & $>15-35$ & 0 & 0 & 0 & 0 \\
\hline T6-600 & 0 & 0 & 0 & 0 & 0 & $>85-<100$ & 0 & 0 & 0 & 0 & $>0-15$ & 0 & 0 & 0 & 0 \\
\hline T6-665 & 0 & $>35.65$ & 0 & 0 & 0 & 0 & 0 & 0 & 0 & 0 & $>15-35$ & 0 & 0 & 0 & $>35-65$ \\
\hline$T 7-5$ & $>35-65$ & 0 & 0 & $>35-65$ & 0 & 0 & 0 & 0 & 0 & 0 & $>0-15$ & 0 & 0 & 0 & 0 \\
\hline$T 7-50$ & $>65-85$ & 0 & 0 & 0 & 0 & 0 & 0 & 0 & 0 & 0 & $>15-35$ & 0 & 0 & 0 & 0 \\
\hline T7.130 & 0 & 0 & 0 & 0 & 0 & 0 & 0 & 0 & 0 & 0 & 0 & 0 & 100 & 0 & 0 \\
\hline $\mathrm{T} 7-160$ & $>35-65$ & 0 & 0 & 0 & 0 & 0 & 0 & 0 & 0 & 0 & $>35-65$ & 0 & 0 & 0 & 0 \\
\hline$T 7-410$ & 0 & 0 & 0 & 0 & 0 & 0 & $>0-15$ & 0 & $>85-<100$ & 0 & $>0-15$ & 0 & 0 & 0 & 0 \\
\hline $\mathrm{T} 7-480$ & 0 & 100 & 0 & 0 & 0 & 0 & 0 & 0 & 0 & 0 & 0 & 0 & 0 & 0 & 0 \\
\hline T7-550 & 100 & 0 & 0 & 0 & 0 & 0 & 0 & 0 & 0 & 0 & 0 & 0 & 0 & 0 & 0 \\
\hline T7-610 & 0 & 0 & 0 & 0 & 0 & 0 & 0 & 0 & 0 & 0 & $>0-15$ & 0 & 0 & 0 & $>85-<100$ \\
\hline
\end{tabular}




\begin{tabular}{|c|c|c|c|c|c|c|c|c|c|c|c|c|c|c|c|}
\hline 0 & 0 & 0 & 0 & 0 & 0 & 0 & 0 & 0 & 0 & 0 & $\mathcal{S E}-\mathcal{S} \mid<$ & $\varsigma_{\mathcal{E}}-\varsigma_{\mathfrak{I}}<$ & $\varsigma 9-\varsigma \varepsilon<$ & 0 & $00 Z-E L L$ \\
\hline 0 & 0 & 0 & 0 & 0 & 0 & 0 & 0 & 0 & 0 & 0 & $\mathrm{SI}^{-0} 0<$ & $\$ 8-\varsigma 9<$ & 0 & 0 & $S Z I-\varepsilon I L$ \\
\hline aN & aN & aN & an & aN & वN & aN & बN & aN & aN & aN & an & aN & aN & aN & $0 \varsigma^{-\varepsilon I L}$ \\
\hline 0 & 0 & $\varsigma 8-\varsigma 9<$ & 0 & 0 & 0 & 0 & 0 & 0 & 0 & SI-0< & 0 & $s I^{-0}<$ & 0 & 0 & $0 \downarrow z-z I L$ \\
\hline aN & aN & aN & aN & aN & aN & वN & an & aN & aN & $\mathrm{aN}_{\mathrm{N}}$ & aN & $\mathrm{aN}$ & an & aN & S9I-ZIL \\
\hline an & aN & aN & बN & aN & aN & $\mathrm{aN}_{\mathrm{N}}$ & बN & aN & aN & aN & an & aN & aN & वN & 00I-ZIL \\
\hline aN & đN & $\mathrm{aN}$ & $\mathrm{dN}$ & an & aN & an & $\mathrm{aN}_{\mathrm{N}}$ & an & aN & aN & aN & an & aN & aN & $\varsigma \tau-\tau I L$ \\
\hline 0 & 0 & 0 & 0 & 0 & 0 & 0 & 0 & 0 & 0 & sI-0< & 0 & $\varsigma 9-\varsigma \mathcal{E}<$ & $\varsigma 9-\varsigma \mathcal{E}<$ & 0 & SLI-IIL \\
\hline 0 & 0 & 0 & 0 & 0 & 0 & 0 & 0 & 0 & 0 & 0 & $\varsigma 8-\varsigma 9<$ & 0 & 0 & $\varsigma \mathcal{E}-\varsigma \mathrm{l}<$ & ${ }_{\mathrm{I}} 00 \mathrm{I}^{-} \mathrm{I} \mathrm{IL}$ \\
\hline 0 & 0 & $S I-0<$ & 0 & $\mathrm{SI}^{-} 0<$ & 0 & 0 & 0 & 0 & 0 & 0 & $\varsigma 9-\varsigma \varepsilon<$ & 0 & 0 & $\mathcal{S E}-\mathcal{S} \mathbf{I}$ & 00I-IIL \\
\hline 0 & 0 & 0 & 0 & $s_{1}-0<$ & 0 & 0 & 0 & 0 & 0 & 0 & 0 & 0 & 0 & $001>-\varsigma 8<$ & SZ-ILL \\
\hline 0 & 0 & 0 & 0 & 0 & 0 & 0 & 0 & 0 & 0 & 0 & 0 & 0 & 0 & $00 \mathrm{I}$ & OSI-0IL \\
\hline 0 & 0 & 0 & 0 & $\varsigma^{-}-0<$ & 0 & 0 & 0 & 0 & 0 & 0 & 0 & 0 & $00 \mathrm{I}>-58<$ & 0 & $00 \mathrm{I}-0 \mathrm{LI}$ \\
\hline 0 & 0 & 0 & 0 & 0 & 0 & 0 & 0 & 0 & 0 & 0 & 0 & 0 & 0 & $00 \mathrm{I}$ & $S z-0 I L$ \\
\hline aN & aN & aN & an & aN & aN & $\mathrm{aN}_{\mathrm{N}}$ & aN & $\mathrm{aN}_{\mathrm{N}}$ & $\mathrm{aN}_{\mathrm{N}}$ & aN & बN & aN & aN & aN & $\varsigma 6 \varepsilon-6 \mathrm{~L}$ \\
\hline 0 & 0 & 0 & 0 & 0 & 0 & 0 & 0 & 0 & 0 & 0 & 0 & 0 & 0 & $00 \mathrm{I}$ & $S \tau \varepsilon^{-}-6 L$ \\
\hline 0 & 0 & 0 & 0 & 0 & 0 & 0 & 0 & 0 & 0 & 0 & 0 & 0 & 0 & $00 \mathrm{I}$ & $\$ S Z-6 \mathrm{LL}$ \\
\hline 0 & 0 & 0 & 0 & 0 & 0 & 0 & 0 & 0 & 0 & 0 & 0 & 0 & 0 & $00 \mathrm{I}$ & $58 \mathrm{I}-6 \mathrm{~L}$ \\
\hline 0 & 0 & 0 & 0 & 0 & 0 & 0 & 0 & 0 & 0 & 0 & 0 & 0 & 0 & 001 & SII-6L. \\
\hline 0 & 0 & 0 & 0 & 0 & 0 & 0 & 0 & 0 & 0 & 0 & 0 & 0 & 0 & $00 \mathrm{I}$ & $\mathcal{S E}-6 \mathrm{~L}$ \\
\hline 0 & 0 & 0 & 0 & 0 & 0 & 0 & 0 & 0 & 0 & 0 & 0 & 0 & 0 & $00 \mathrm{I}$ & $S I \cdot 6 \mathrm{I}$ \\
\hline 0 & SI-0< & 0 & 0 & $S_{I}-0<$ & 0 & 0 & 0 & $58-\varsigma 9<$ & 0 & 0 & 0 & 0 & 0 & 0 & $06 t-8 L$ \\
\hline 0 & $\mathcal{S E}-\mathcal{S I}<$ & 0 & 0 & 0 & $\varsigma \mathcal{E}-\varsigma \mathfrak{L}<$ & 0 & 0 & $\varsigma 9-\varsigma \mathcal{E}<$ & 0 & 0 & 0 & 0 & 0 & 0 & $02 t-8 \mathrm{~L}$ \\
\hline 0 & 0 & 0 & 0 & 0 & 0 & 0 & 0 & 0 & 0 & 0 & 0 & 0 & 0 & $00 \mathrm{I}$ & 0SE-8L \\
\hline 0 & 0 & 0 & 0 & 0 & 0 & 0 & 0 & 0 & 0 & 0 & 0 & 0 & 0 & $00 \mathrm{I}$ & $082-8 \mathrm{~L}$ \\
\hline 0 & 0 & 0 & 0 & 0 & 0 & 0 & 0 & 0 & 0 & 0 & 0 & 0 & 0 & 001 & $0 I Z-8 \mathbf{L}$ \\
\hline 0 & 0 & 0 & 0 & 0 & 0 & 0 & 0 & 0 & 0 & 0 & 0 & 0 & 0 & $00 \mathrm{I}$ & $00 \mathrm{I}-8 \mathrm{~L}$ \\
\hline 0 & 0 & 0 & 0 &  & 0 & 0 & 0 & 0 & 0 & 0 & 0 & 0 & 0 & 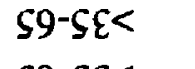 & $0 L-8 \mathbf{L}$ \\
\hline 0 & 0 & 0 & 0 & 0 & 0 & 0 & 0 & 0 & 0 & 0 & 0 & 0 & $\varsigma 9-\varsigma \mathcal{E}<$ & S9-SE< & S-8I \\
\hline 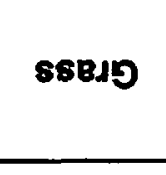 & $\begin{array}{l}\text { (oseq) } \\
\text { əuurd }\end{array}$ & YOEגM & $\begin{array}{l}\text { !p」eja6 } \\
\text { snounf }\end{array}$ & $\begin{array}{c}\text { suəj!n } \\
\text {-jaduas } \\
\text { ofep!los }\end{array}$ & $\begin{array}{l}\text { sueosed } \\
\text {-ndind } \\
\text { eoyon/d }\end{array}$ & $\begin{array}{c}\cdot d s \\
\text { eyd/1 }\end{array}$ & $\begin{array}{c}\text { Bezdouna } \\
\text { E!U } \\
\text { - Jos!les }\end{array}$ & $\begin{array}{c}\text { sn! } \\
\text { snqos } \\
\text { sndj!os }\end{array}$ & $\begin{array}{l}\text { sopjouns } \\
\text {-oufo } \\
\text { Bu!neds }\end{array}$ & $\begin{array}{l}\text { suevs } \\
\text {-OInAy } \\
\text { EAI }\end{array}$ & $\begin{array}{c}\text { EqEo!ds } \\
\text { s!!yo!ns!a }\end{array}$ & 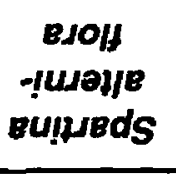 & $\begin{array}{l}\text { suajed } \\
\text { eu!pleds }\end{array}$ & $\begin{array}{l}\text { s!jejusne } \\
\text { soỵu } \\
\text {-6esyd } \\
\end{array}$ & $\begin{array}{l}\text { 'on } \\
\text { ẹ̣s 6u!j|d } \\
\text {-wes } \\
\text { pur }\end{array}$ \\
\hline \multicolumn{15}{|c|}{ 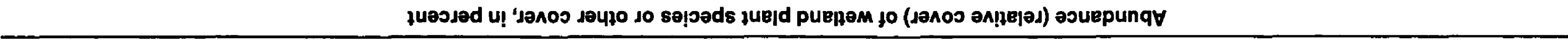 } & posue. \\
\hline
\end{tabular}


Table 1. Abundance (relative cover) of wetland plants at sampling sites along transects, Inner Namskaket Marsh, Cape Cod, Massachusetts, August and September 1995-Continued

\begin{tabular}{|c|c|c|c|c|c|c|c|c|c|c|c|c|c|c|c|}
\hline \multirow{2}{*}{$\begin{array}{c}\text { Transect } \\
\text { and } \\
\text { sam- } \\
\text { pling site } \\
\text { No. }\end{array}$} & \multicolumn{15}{|c|}{ Abundance (relative cover) of wetland plant species or other cover, in percent } \\
\hline & $\begin{array}{c}\text { Phrag- } \\
\text { mites } \\
\text { australis }\end{array}$ & $\begin{array}{c}\text { Spartina } \\
\text { patens }\end{array}$ & $\begin{array}{c}\text { Spartina } \\
\text { alterni- } \\
\text { flora }\end{array}$ & $\begin{array}{c}\text { Distichlis } \\
\text { spicata }\end{array}$ & $\begin{array}{l}\text { Iva } \\
\text { trute- } \\
\text { scens }\end{array}$ & $\begin{array}{l}\text { Spartina } \\
\text { cyno- } \\
\text { suroides }\end{array}$ & $\begin{array}{c}\text { Scirpus } \\
\text { robus- } \\
\text { tus }\end{array}$ & $\begin{array}{l}\text { Salicor- } \\
\text { nia } \\
\text { europaea }\end{array}$ & $\begin{array}{c}\text { Typha } \\
\text { sp. }\end{array}$ & $\begin{array}{l}\text { Pluchea } \\
\text { purpu- } \\
\text { rascens }\end{array}$ & $\begin{array}{c}\text { Solidago } \\
\text { semper- } \\
\text { virens }\end{array}$ & $\begin{array}{l}\text { Juncus } \\
\text { gerardi }\end{array}$ & Wrack & $\begin{array}{l}\text { Panne } \\
\text { (bare) }\end{array}$ & Grass \\
\hline$\overline{\mathrm{T} 14-0}$ & $>15-35$ & 0 & 0 & $\overline{0}$ & 0 & 0 & 0 & $\overline{0}$ & $\overline{0}$ & $\overline{0}$ & $>15-35$ & $\overline{0}$ & 0 & $\overline{0}$ & ${ }^{2}>35-65$ \\
\hline T14-25 & $>35-65$ & 0 & 0 & 0 & 0 & 0 & 0 & 0 & 0 & 0 & $>15-35$ & 0 & 0 & 0 & ${ }^{3}>15-35$ \\
\hline $\mathrm{T} 14-100$ & $>35-65$ & 0 & 0 & 0 & $>0-15$ & 0 & $>0-15$ & 0 & 0 & 0 & 0 & 0 & 0 & $>35-65$ & 0 \\
\hline $\mathrm{T} 14-100^{1}$ & $>85-<100$ & 0 & 0 & 0 & $>0-15$ & 0 & $>0-15$ & 0 & 0 & 0 & 0 & 0 & 0 & $>0-15$ & 0 \\
\hline T14-175 & ND & ND & ND & ND & ND & ND & ND & ND & ND & ND & ND & ND & ND & ND & ND \\
\hline $\mathrm{T} 14-215^{4}$ & 0 & $>35-65$ & 0 & $>35-65$ & 0 & 0 & 0 & 0 & 0 & 0 & 0 & $>0-15$ & 0 & 0 & 0 \\
\hline $\mathrm{T} 14-275$ & ND & ND & ND & ND & ND & ND & ND & ND & $\mathrm{ND}$ & ND & ND & ND & ND & ND & ND \\
\hline $\mathrm{T} 15-25$ & $>15-35$ & 0 & 0 & 0 & 0 & 0 & 0 & $>15-35$ & 0 & $>15-35$ & 0 & 0 & 0 & $>15-35$ & 0 \\
\hline $\mathrm{T} 15-25^{1}$ & 100 & 0 & 0 & 0 & 0 & 0 & 0 & 0 & 0 & 0 & 0 & 0 & 0 & 0 & 0 \\
\hline $\mathrm{T} 15-100$ & $>15-35$ & 0 & 0 & 0 & 0 & 0 & 0 & 0 & 0 & $>0-15$ & $>15-35$ & 0 & 0 & $>15-35$ & 0 \\
\hline $\mathrm{T} 15-100^{1}$ & 0 & $>85-<100$ & 0 & $>0-15$ & 0 & 0 & 0 & 0 & 0 & 0 & 0 & 0 & 0 & 0 & 0 \\
\hline T15-175 & $>35-65$ & 0 & 0 & 0 & 0 & 0 & 0 & 0 & 0 & 0 & $>0-15$ & 0 & 0 & $>35-65$ & 0 \\
\hline $\mathrm{T} 15-175^{1}$ & $>35-65$ & 0 & 0 & $>0-15$ & 0 & 0 & 0 & 0 & 0 & 0 & $>15-35$ & 0 & 0 & 0 & ${ }^{5}>15-35$ \\
\hline T15-250 & 0 & $>35-65$ & $>15-35$ & $>0-15$ & 0 & 0 & 0 & 0 & 0 & 0 & 0 & 0 & 0 & 0 & 0 \\
\hline $\mathrm{T} 15-250^{1}$ & 0 & $>85-<100$ & $>0.15$ & $>0-15$ & 0 & 0 & 0 & 0 & 0 & 0 & 0 & 0 & 0 & 0 & 0 \\
\hline T15-275 & 0 & 0 & 100 & 0 & 0 & 0 & 0 & 0 & 0 & 0 & 0 & 0 & 0 & 0 & 0 \\
\hline T16-25 & 0 & 0 & 0 & 0 & 0 & 0 & 0 & 0 & 0 & 0 & 0 & 0 & 100 & 0 & 0 \\
\hline T16-100 & 0 & 0 & 0 & $>15-35$ & 0 & 0 & 0 & 0 & 0 & 0 & 0 & 0 & $>65-85$ & 0 & 0 \\
\hline $\mathrm{T} 16-100^{1}$ & 0 & $>0-15$ & $>0-15$ & $>85-<100$ & 0 & 0 & 0 & 0 & 0 & 0 & 0 & 0 & 0 & 0 & 0 \\
\hline T16-175 & 0 & $>85-<100$ & $>0-15$ & 0 & 0 & 0 & 0 & 0 & 0 & 0 & 0 & 0 & 0 & 0 & 0 \\
\hline T16-240 & 0 & $>85-<100$ & $>0-15$ & 0 & 0 & 0 & 0 & 0 & 0 & 0 & 0 & 0 & 0 & 0 & 0 \\
\hline T16-340 & 0 & 100 & 0 & 0 & 0 & 0 & 0 & 0 & 0 & 0 & 0 & 0 & 0 & 0 & 0 \\
\hline
\end{tabular}

1 Abundance determined within approximately $3 \mathrm{~m}$ of sampling site.

2 Includes 25 percent Hibiscus moschentos and 20 percent Rosa sp.

${ }^{3}$ Includes 25 percent $H$. moschentos.

${ }^{4}$ Sampling site located at 215 feet from marsh/upland boundary along Transect 14 between T14-175 and T14-275; not shown on figure 2

5 Includes 30 percent $H$. moschentos. 
Table 2. Measured areal coverage of wetland plants and plant associations, Inner Namskaket Marsh, Cape Cod, Massachusetts, August through October 1995

[Mapped vegetated area excludes undetermined, creek, and upland areas within the boundaries of the study area]

\begin{tabular}{|c|c|c|}
\hline \multirow{2}{*}{ Plant species } & \multicolumn{2}{|c|}{ Mapped vegetated area } \\
\hline & Hectares & Percentage \\
\hline \multicolumn{3}{|c|}{ Inner Namskaket Marsh } \\
\hline Phragmites australis................. & 2.16 & 38.3 \\
\hline  & .83 & 14.7 \\
\hline Spartina alterniflora ................. & .43 & 7.6 \\
\hline 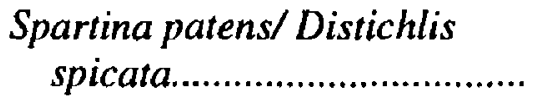 & .38 & 6.7 \\
\hline 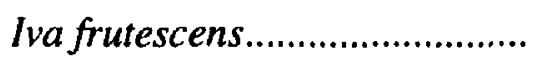 & .30 & 5.4 \\
\hline Distichlis spicata ..................... & .23 & 4.1 \\
\hline Spartina cynosuroides .............. & .22 & 3.9 \\
\hline $\begin{array}{l}\text { Spartina patens/ Spartina } \\
\text { cynosuroides } . . . . . . . . . . . . . . . . . . . . . . . .\end{array}$ & .14 & 2.5 \\
\hline  & .084 & 1.5 \\
\hline  & .055 & 1.0 \\
\hline Salicornia europaea ................. & .020 & .35 \\
\hline 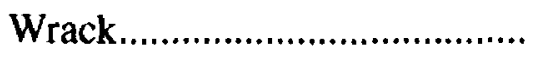 & .78 & 13.7 \\
\hline Panne & .022 & .40 \\
\hline Creek & .53 & - \\
\hline 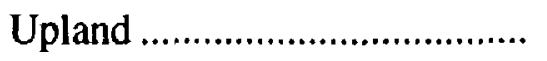 & .33 & -- \\
\hline  & .73 & -- \\
\hline \multicolumn{3}{|c|}{ Hurley's Bog } \\
\hline Phragmites australis................ & 0.27 & 21.1 \\
\hline Iva frutescens........................... & .024 & 1.9 \\
\hline 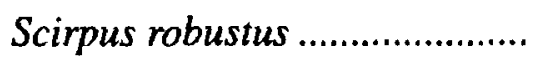 & .023 & 1.8 \\
\hline $\begin{array}{l}\text { Solidago sempervirens/ } \\
\quad \text { Spartina patens..................... }\end{array}$ & .020 & 1.6 \\
\hline $\begin{array}{l}\text { Phragmites australis/ } \\
\quad \text { Solidago sempervirens......... }\end{array}$ & .009 & .73 \\
\hline  & .92 & 73.0 \\
\hline Creek & .022 & -- \\
\hline
\end{tabular}

In the late summer/early autumn 1995, wrack deposits covered almost 15 percent of the mapped area (fig. 5 and table 2). These deposits were dynamic and moved with exceptionally high tides; for example, much of the wrack-covered area near transect T5 (figs. 3 and 5) was bare in the 1996 growing season. The effect of wrack on underlying vegetation varies among species and with the duration of burial (Hartman, 1988). Distichlis spicata is less vulnerable to short-term burial than
$S$. patens and, if killed, will recolonize more rapidly (Bertness and Ellison, 1987). The distributions of Distichlis spicata and S. patens in areas of the marsh where wrack accumulates likely have been affected by previous deposition and some areas where Distichlis spicata predominates (for example, near T2-260, figs. 3 and 5) may be recovering bare patches (Bertness and others, 1992). The distribution of Salicornia europaea, which can rapidly colonize bare patches and tolerates the high salinity of pannes, also is controlled by physical disturbance in the study area. Salicornia europaea is an invasive species that is typically displaced by Distichlis spicata (Ellison, 1987; Bertness and Ellison, 1987). Ice scouring and winter storms are other natural disturbances that may contribute to the patchy distribution of plants in the marsh. Finally, the effects of mosquito ditching during the 1930's on plant distributions may still be evidenced by the occurrence of Iva frutescens (not mapped separately) along the two narrow, linear ditches in the eastern part of Inner Namskaket marsh. Iva grows on banks and spoils from mosquito ditching as well as along upland borders (Shisler and others, 1978; Nixon, 1982; Tiner, 1987).

The dominant wetland plant in Hurley's Bog is Phragmites australis, which covers about 80 percent of the open, herbaceous area (fig. 5 and table 2). Iva frutescens, Solidago sempervirens, and S. patens occur along the main creek from the culvert, and Scirpus robustus surrounds an adjacent, poorly defined creek that drains toward the embankment. Teal and Giblin (1983) also noted Scirpus, Juncus, Spartina patens, and Iva frutescens in the marsh center, which experiences seawater flooding, and Phragmites, Typha, and shrubs of red maple and sweet pepperbush at the edges of the herbaceous or marshy area. Surrounding the open, herbaceous area is a shrub oak/maple wet woodland, which is not penetrated by creeks or tidal flows (fig. 5). This wet woodland is flooded only by seasonal, high freshwater inflows. The predominance of brackish to freshwater wetland species (especially Phragmites) and limited area of herbaceous plants in Hurley's Bog likely reflect the restricted tidal flow into the bog, which before construction of the railroad embankment in the late 1800's was freely connected with Inner Namskaket Marsh (Teal and Giblin, 1983). The linearity and angular junctions of the creeks in the bog suggest that the creeks also were altered at some time in the past. 
Aboveground biomass, stem density, and leafnitrogen content provide quantitative measures of the existing plant community from which to assess potential changes caused by increased nitrogen loading. Results of the baseline survey of selected sampling sites in Inner Namskaket Marsh are presented in tables 3 and 4 (see figs. 2 and 3 for sampling sites). Aboveground biomass at the peak of the growing season is an indicator of production that is related to annual productivity (Linthurst and Reimold, 1978; Nixon, 1982). This measure underestimates total primary production because it does not include leaf turnover and belowground production, which may be as much as four times aboveground end-of-season biomass in high marshes (Valiela and others, 1976; Good and others, 1982). However, peak aboveground biomass can be used to distinguish areas of high and low productivity and is stable from year to year (Teal and Howes, 1996); thus, peak aboveground biomass is a good indicator of potential changes in the productivity of the salt marsh vegetation. Aboveground biomass measurements in the marsh (table 3) are similar to reported values of aboveground biomass at the end of the growing season of 300 to $1,000 \mathrm{~g}$ of dry weight per $\mathrm{m}^{2} / \mathrm{yr}$ for $S$. patens, 250 to $510 \mathrm{~g}$ of dry weight per $\mathrm{m}^{2} / \mathrm{yr}$ for short-form $S$. alterniflora, and 440 to $680 \mathrm{~g}$ of dry weight per $\mathrm{m}^{2} / \mathrm{yr}$ for S. patens/D. spicata mixes in high-marsh areas of southern New England (Nixon, 1982, and references therein); $472 \mathrm{~g}$ of dry weight per $\mathrm{m}^{2} / \mathrm{yr}$ for short-form $S$. alterniflora in nearby Nauset Marsh, Eastham and Orleans, Massachusetts (Roman and others, 1990); and 632 and $424 \mathrm{~g}$ of dry weight per $\mathrm{m}^{2} / \mathrm{yr}$ for $S$. patens and

Table 3. Wetland plant stem density and aboveground biomass at selected sampling sites, Inner Namskaket Marsh, Cape Cod, Massachusetts, August and September 1995

[See figure 2 for locations of sampling sites; stem density and aboveground biomass include live stems only]

\begin{tabular}{|c|c|c|c|c|c|c|c|}
\hline $\begin{array}{c}\text { Transect } \\
\text { and } \\
\text { sampling } \\
\text { site No. }\end{array}$ & $\begin{array}{l}\text { Wetland plant } \\
\text { species }\end{array}$ & $\begin{array}{c}\text { Stem } \\
\text { density } \\
\text { (stems per } \\
\text { square } \\
\text { meter) }\end{array}$ & $\begin{array}{l}\text { Aboveground } \\
\text { biomass } \\
\text { (grams dry } \\
\text { weight per } \\
\text { square meter) }\end{array}$ & $\begin{array}{l}\text { Transect } \\
\text { and } \\
\text { sampling } \\
\text { site No. }\end{array}$ & $\begin{array}{l}\text { Wetland plant } \\
\text { species }\end{array}$ & $\begin{array}{c}\text { Stem } \\
\text { density } \\
\text { (stems per } \\
\text { square } \\
\text { meter) }\end{array}$ & $\begin{array}{l}\text { Aboveground } \\
\text { biomass } \\
\text { (grams dry } \\
\text { weight per } \\
\text { square meter) }\end{array}$ \\
\hline \multirow[t]{3}{*}{$\mathrm{T} 2-560^{1}$} & Distichlis spicata & 520 & 230 & T3-310 & Spartina alterniflora & 310 & 680 \\
\hline & Spartina patens & 4,300 & 340 & T3-570 & Juncus gerardi & 1,390 & 120 \\
\hline & Total .............. & 4,820 & 570 & $12-580$ & Solidago sempervirens & 20 & 20 \\
\hline \multirow[t]{3}{*}{$\mathrm{T} 2-660^{1}$} & Distichlis spicata & 5,120 & 690 & & Spartina patens & 3,310 & 250 \\
\hline & Salicomia europaea & 190 & 60 & & 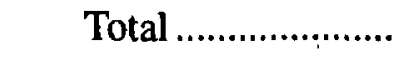 & 4,720 & 390 \\
\hline & Spartina patens & $\begin{array}{r}630 \\
5940\end{array}$ & $\begin{array}{r}80 \\
880\end{array}$ & $\mathrm{~T} 7-480$ & Spartina patens & 8,990 & 1,050 \\
\hline \multirow{3}{*}{$\mathrm{T} 2-705$} & & & & $\mathrm{~T} 13.125$ & Spartina alterniflora & 310 & 330 \\
\hline & Distichlis spicata & 550 & 130 & & Spartina patens & 470 & 60 \\
\hline & $\begin{array}{l}\text { Spartina patens } \\
\text { Total } . . . \ldots \ldots \ldots \ldots . . . . .\end{array}$ & $\begin{array}{l}4,000 \\
4,550\end{array}$ & $\begin{array}{l}360 \\
490\end{array}$ & & Total & 780 & 390 \\
\hline \multirow{4}{*}{$\mathrm{T} 3-45^{1}$} & Dictichlis snicata & 960 & 310 & T13-200 & Distichlis spicata & 240 & 90 \\
\hline & Distichis spicata & $\begin{array}{r}900 \\
40\end{array}$ & 300 & & Spartina alterniflora & 80 & 30 \\
\hline & Phragmites australis & $\begin{array}{r}40 \\
1000\end{array}$ & $\begin{array}{l}200 \\
510\end{array}$ & & Spartina patens & 5,580 & 680 \\
\hline & 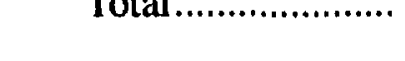 & 1,000 & 310 & & Total ......................... & 5,900 & 810 \\
\hline T3-170 & Spartina patens & 8,950 & 890 & T14-275 & Distichlis spicata & 150 & 80 \\
\hline \multirow[t]{3}{*}{ T3-240 } & Phragmites australis & 230 & 720 & & Spartina patens & 5,860 & 500 \\
\hline & Pluchea purpurascens & 50 & 40 & & Total ......................... & 6,010 & 580 \\
\hline & Spartina patens & $\begin{array}{l}1,420 \\
1700\end{array}$ & $\begin{array}{l}130 \\
890\end{array}$ & T15-275 & Spartina alterniflora & 260 & 610 \\
\hline \multirow{4}{*}{ T3-300 } & tichlis snicata & 380 & 90 & $\mathrm{~T} 16-340^{1}$ & Spartina patens & 8,400 & 640 \\
\hline & Salicomia europaea & 260 & 350 & & & & \\
\hline & Spartina patens & 400 & 80 & & & & \\
\hline & Total ....................... & 1,040 & 520 & & & & \\
\hline
\end{tabular}

\footnotetext{
${ }^{1}$ One replicate only
} 
Table 4. Wetland plant stem density and leaf-nitrogen content at selected sampling sites, Inner Namskaket Marsh, Cape Cod, Massachusetts, August 7, 1996

[Site No.: See figure 3 for locations of sampling sites. Stem density includes live stems only. Height and leaf-nitrogen content were measured for samples of primary plant species. $\mathrm{m}^{2}$, square meter; $\mathrm{cm}$, centimeter; -- , not present; $\mathrm{ND}$, not determined]

\begin{tabular}{|c|c|c|c|c|c|c|c|c|c|}
\hline \multirow[t]{2}{*}{ Site No. } & \multicolumn{2}{|c|}{ Primary plant species } & \multicolumn{2}{|c|}{$\begin{array}{l}\text { Secondary plant species } \\
\text { (if present) }\end{array}$} & \multirow{2}{*}{$\begin{array}{l}\text { Total stem } \\
\text { density } \\
\text { (stems } / \mathrm{m}^{2} \text { ) }\end{array}$} & \multicolumn{2}{|c|}{$\begin{array}{l}\text { Height of } \\
\text { sampled plants } \\
\text { (cm) }\end{array}$} & \multicolumn{2}{|c|}{$\begin{array}{l}\text { Leaf-nitrogen content of } \\
\text { sampled plants } \\
\text { (percentage of } \\
\text { dry weight) }\end{array}$} \\
\hline & Species & $\begin{array}{l}\text { Stem density } \\
\left(\text { stems } / \mathbf{m}^{2}\right)\end{array}$ & Species & $\begin{array}{l}\text { Stem density } \\
\left(\mathbf{s t e m s} / \mathbf{m}^{2}\right)\end{array}$ & & Mean & $\begin{array}{l}\text { Standard } \\
\text { deviation }\end{array}$ & Mean & $\begin{array}{l}\text { Standard } \\
\text { deviation }\end{array}$ \\
\hline 1 & $\begin{array}{c}\text { Phragmites } \\
\text { australis }\end{array}$ & 32 & -. & -- & 32 & 128 & 7.0 & 2.91 & 0.13 \\
\hline 2 & $\begin{array}{l}\text { Scirpus } \\
\quad \text { robustus }\end{array}$ & 74 & $\begin{array}{c}\text { Phragmites } \\
\text { australis }\end{array}$ & 40 & 114 & 52 & 7.5 & 2.03 & .06 \\
\hline 3 & $\begin{array}{c}\text { Phragmites } \\
\text { australis }\end{array}$ & 52 & .. & -. & 52 & 111 & 4.4 & 3.44 & .03 \\
\hline 4 & $\begin{array}{c}\text { Phragmites } \\
\text { australis }\end{array}$ & 68 & -. & -- & 68 & 130 & 4.7 & 3.34 & .12 \\
\hline 5 & $\begin{array}{c}\text { Phragmites } \\
\text { australis }\end{array}$ & 36 & $\begin{array}{l}\text { Scirpus } \\
\quad \text { robustus }\end{array}$ & 2 & 38 & 90 & 8.0 & 2.92 & .09 \\
\hline 6 & $\begin{array}{l}\text { Scirpus } \\
\quad \text { robustus }\end{array}$ & 80 & $\begin{array}{c}\text { Phragmites } \\
\text { australis }\end{array}$ & ND & 80 & 71 & 2.5 & 1.96 & .08 \\
\hline 7 & $\begin{array}{c}\text { Phragmites } \\
\text { australis }\end{array}$ & 32 & $\begin{array}{l}\text { Scirpus } \\
\quad \text { robustus }\end{array}$ & 4 & 36 & 108 & 17 & 3.26 & .03 \\
\hline 8 & $\begin{array}{c}\text { Phragmites } \\
\text { australis }\end{array}$ & 46 & -- & -- & 46 & 120 & 14 & 3.89 & .24 \\
\hline 9 & $\begin{array}{c}\text { Phragmites } \\
\text { australis }\end{array}$ & 70 & .. & -- & 70 & 76 & 13 & 3.12 & .24 \\
\hline 10 & $\begin{array}{l}\text { Phragmites } \\
\text { australis }\end{array}$ & 82 & -- & -- & 82 & 79 & 11 & 3.10 & .01 \\
\hline 11 & $\begin{array}{l}\text { Scirpus } \\
\text { robustus }\end{array}$ & 42 & $\begin{array}{c}\text { Phragmites } \\
\text { australis }\end{array}$ & 20 & 62 & 57 & 5.7 & 1.80 & .10 \\
\hline 12 & $\begin{array}{l}\text { Phragmites } \\
\text { australis }\end{array}$ & 52 & $\begin{array}{l}\text { Scirpus } \\
\quad \text { robustus }\end{array}$ & 8 & 60 & 70 & 9.3 & 3.08 & .10 \\
\hline 13 & $\begin{array}{c}\text { Phragmites } \\
\text { australis }\end{array}$ & 64 & $\begin{array}{l}\text { Scirpus } \\
\quad \text { robustus }\end{array}$ & 14 & 78 & 61 & 10 & 2.82 & .18 \\
\hline 14 & $\begin{array}{l}\text { Scirpus } \\
\text { robustus }\end{array}$ & ND & $\begin{array}{c}\text { Phragmites } \\
\text { australis }\end{array}$ & ND & ND & 42 & 1.7 & 1.69 & .12 \\
\hline 15 & $\begin{array}{c}\text { Phragmites } \\
\text { australis }\end{array}$ & 60 & -- & -- & 60 & 99 & 8.3 & 3.07 & .10 \\
\hline
\end{tabular}

low-marsh S. alterniflora, respectively, in Great Sippewissett Marsh, Falmouth, Massachusetts (Valiela and others, 1976).

Leaf-nitrogen samples were taken in mid-July, near the time of peak biomass. Leaf-nitrogen content changes during the growing season, reaching maximum values in the spring for many species (Roman and Daiber, 1984). However, like aboveground biomass, leaf-nitrogen content is relatively stable from year to year at similar times during the growing season, and differences in leaf-nitrogen content among stands of the same species at the similar times during the growing season can be used to assess nitrogen availability or loading (Vince and others, 1981). Thus, the measured values represent baseline measurements to which potential future changes could be referenced with samples taken near the peak of the growing season. The sampling sites were distributed along the eastern marsh/ upland boundary, where ground-water seepage is greatest (Weiskel and others, 1996) in order to document leaf-nitrogen content in those vegetated areas with the greatest potential for exposure to increased nitrogen loading (fig. 3). Leaf-nitrogen content averaged $3.18 \pm 0.30( \pm 1$ standard deviation, $n=11)$ and $1.87 \pm 0.15( \pm 1$ standard deviation, $n=4)$ percentage of dry weight in Phragmites australis and Scirpus robustus samples, respectively. 


\section{Effect of Nitrogen Enrichment}

The nitrogen-enrichment experiment was designed to simulate the discharge of effluentcontaminated ground water on the two dominant plant associations in Inner Namskaket Marsh, Phragmites australis and S. patens/S. alterniflora. Nitrogen was applied as dissolved nitrate, which is the dominant form of nitrogen in the plume of contaminated ground water that is available to plants. The fertilization rate was based on nitrate concentrations in the center of the plume and on an estimated area of discharge determined from its dimensions (DeSimone and others, 1996). Nitrogen was applied at the marsh surface because it is expected that, if effluent-contaminated ground water discharges to the marsh, it will enter through the seepage zones at the marsh boundary or through the creek bottoms rather than directly through the thick, low-permeability peat underlying most of the marsh (Howes and others, 1996; Weiskel and others, 1997) Thus, effiuent nitrogen would be expected to reach most marsh vegetation through overland flow. The experimental application rate was intended to simulate discharge of the effluent plume in the marsh and represents a maximum estimated nitrogen loading rate associated with plume discharge.

After the first year of fertilization in Phragmites plots, stem density and aboveground biomass showed average increases of 66 and 21 percent, respectively (table 5 and fig. 7A); the effects of the second year of fertilization were similar (28 and 43 percent, respectively). Leaf-nitrogen content after the second year increased about 26 percent under fertilization. These increases suggest a modest enhancement of Phragmites growth and potential food quality to grazing organisms. Detrimental effects of nitrate additions were not detected. However, the effects of the second year of fertilization were associated with a four- to five-fold increase in stem density and a general increase in aboveground biomass relative to 1995 values in control and fertilized plots. These increases most likely resulted from the disturbance to the plots caused by the 1995 harvesting, because the growth of new Phragmites stems is stimulated when stems are broken (Haslam, 1969).

In contrast to the effect on Phragmites growth, the primary effect of nitrogen enrichment on Spartina was a substantial shift in species composition. Whereas control plots were nearly monospecific $S$. patens (96 to 99 percent of stems and 70 to 90 percent of total biomass) in 1995 and 1996, S. alterniflora increased under fertilization to represent nearly one-half of total biom- ass by the end of the first growing season (table 5 and fig. $7 B$ ). This species shift was readily visible in the field (fig. 8). At the end of the second year of fertilization, S. alterniflora plants outnumbered $S$. patens and represented more than 90 percent of the total biomass in the fertilized plots. S. alterniflora apparently can grow well in the high marsh environment, but is "out-competed" by $S$. patens under natural conditions (Bertness and Ellison, 1987; Bertness, 1992). Thus, nitrogen addition in Inner Namskaket Marsh appeared to alter the competitive balance between $S$. patens and $S$. alterniflora. The observed shift probably did not result from the increased water addition because both control and fertilized plots received identical volumes of creek water.

Results of the nitrogen fertilization experiment in Spartina plots at Namskaket are similar in some respects to results of experiments in Great Sippewissett Marsh in Falmouth, Cape Cod, in which nitrogen addition resulted in a shift from short-form to tall-form S. alterniflora (Valiela and others, 1985). This is suggested by the mean height of the tallest $S$. alterniflora stems in fertilized plot $2(118 \mathrm{~cm})$ as compared to the control plots $(54$ and $63 \mathrm{~cm}$ ) and fertilized plot $1(50$ $\mathrm{cm}$; table 5). Nitrogen fertilization experiments in Great Sippewissett Marsh in Falmouth, Cape Cod, also resulted in species changes, from $S$. alterniflora to Distichlis in the high marsh at the highest levels of fertilization (Valiela and others, 1985); other species shifts included $S$. patens to $S$. alterniflora but these were not consistently observed (J. M. Teal, oral commun., 1997). Differences in tidal range and salinity between Namskaket and Great Sippewissett marshes may have contributed to these and other differing results. Finally, nitrogen fertilization in Great Sippewissett Marsh resulted in increases in above- and belowground production of $S$. patens and Distichlis, and S. alterniflora that changed over time periods of several years (Valiela and others, 1976; Valiela and others, 1985; Teal, 1986); increases in aboveground biomass from nitrogen addition also have been demonstrated in several other studies (for example, Smart and Barko, 1980, S. alterniflora and Distichlis spicata). At Namskaket, the primary effect of nitrogen addition to the fertilized Spartina plots was the shift in dominant species rather than a major increase in aboveground standing crop during the two growing seasons of the experiment (table 5). Increased production or further species changes might have occurred under continued fertilization and could result from long-term increased nitrogen availability to these plant associations in the marsh. 
Table 5. Stem density, aboveground biomass, and leaf-nitrogen content by species in experimental (fertilized) and control plots of Phragmites australis and Spartina patens/Spartina altemiflora, Inner Namskaket Marsh, Cape Cod, Massachusetts, September 1995 and September 1996

[See figures 3 and 6 for plot locations. Stem density and aboveground biomass include live stems only. Leaf-nitrogen content measured July 23, 1996. Values are means from two replicate subsamples per plot unless otherwise noted; --, not determined]

\begin{tabular}{|c|c|c|c|c|c|c|c|c|c|c|c|c|c|c|c|c|}
\hline \multirow{3}{*}{ Species } & \multicolumn{4}{|c|}{$\begin{array}{l}\text { Stem density } \\
\text { (stems per square meter) }\end{array}$} & \multicolumn{4}{|c|}{$\begin{array}{l}\text { Aboveground biomass } \\
\text { (grams dry weight } \\
\text { per square meter) }\end{array}$} & \multicolumn{4}{|c|}{$\begin{array}{l}\text { Mean height (standard deviation) of } \\
10 \text { taliest stems per plot } \\
\text { (centimeters) }\end{array}$} & \multicolumn{4}{|c|}{$\begin{array}{l}\text { Leaf-nitrogen content } \\
\text { (percentage of dry weight) }\end{array}$} \\
\hline & \multicolumn{2}{|c|}{ Piot 1} & \multicolumn{2}{|c|}{ Plot 2} & \multicolumn{2}{|c|}{ Plot 1} & \multicolumn{2}{|c|}{ Plot 2} & \multicolumn{2}{|c|}{ Plot 1} & \multicolumn{2}{|c|}{ Plot 2} & \multicolumn{2}{|c|}{ Plot 1} & \multicolumn{2}{|c|}{ Plot 2} \\
\hline & c & $\mathbf{F}$ & $\mathbf{C}$ & $\mathbf{F}$ & C & $\mathbf{F}$ & C & $\mathbf{F}$ & C & $\mathbf{F}$ & C & $\mathbf{F}$ & C & $\mathbf{F}$ & C & $\mathbf{F}$ \\
\hline \multicolumn{17}{|c|}{ Phragmites australis Plots } \\
\hline \multicolumn{17}{|l|}{ September 1995} \\
\hline $\begin{array}{l}\text { Phragmites } \\
\quad \text { australis.................... }\end{array}$ & 21 & 34 & 17 & 29 & 429 & 489 & 348 & 454 & $168(30)$ & $183(19)$ & ${ }^{1} 163(25)$ & $176(23)$ & -- & - & -- & -- \\
\hline Solidago sp................ & 2 & 4 & 4 & 4 & -- & -- & $\cdots$ & -- & -- & & -- & -. & -- & - & -- & -- \\
\hline Dead .............................. & -- & -- & -- & - & 17 & 22 & 13 & 23 & -- & -- & -- & -- & -- & - & -- & -- \\
\hline 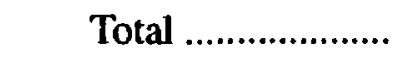 & 23 & 38 & 21 & 33 & 446 & 511 & 361 & 477 & & & & & & & & \\
\hline \multicolumn{17}{|l|}{ September 1996} \\
\hline Phragmites & & & & & & & & & & & & & & & & \\
\hline australis..................... & 107 & 149 & 124 & 146 & 374 & 658 & 570 & 688 & $185(10)$ & $199(12)$ & $203(6)$ & $225(9)$ & 2.49 & 2.87 & 2.55 & 3.48 \\
\hline Solidago sp................ & 3 & 1 & 13 & 0 & 11 & 3 & 49 & 0 & - & -- & -- & -- & $\cdots$ & -- & -- & -- \\
\hline Dead ............................. & -- & -- & - & -- & 27 & 27 & 13 & 45 & -- & -- & -- & -- & -- & -- & -- & -- \\
\hline Total .......................... & 110 & 150 & 137 & 146 & 412 & 688 & 632 & 733 & & & & & & & & \\
\hline \multicolumn{17}{|c|}{ Spartina patens/Spartina alterniflora Plots } \\
\hline \multicolumn{17}{|l|}{ September 1995} \\
\hline Spartina patens....... & 5,050 & 5,400 & 5,200 & 4,175 & 631 & 363 & 574 & 306 & $59(2)$ & $60(5)$ & $54(3)$ & $53(3)$ & -- & -- & -- & -- \\
\hline Spartina alterniflora & 85 & 185 & 215 & 170 & 55 & 304 & 208 & 339 & $247(2)$ & $67(5)$ & $54(4)$ & $63(3)$ & -- & -- & -- & -- \\
\hline Distichlis spicata..... & 150 & 0 & 0 & 0 & 50 & -- & -- & -- & $58(2)$ & -- & -- & -- & -- & -- & -- & $\cdots$ \\
\hline 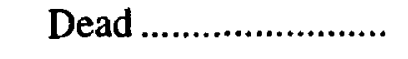 & -- & -- & -- & -- & 40 & 103 & 60 & 74 & -- & -- & -- & -- & -- & -- & -- & -- \\
\hline Total ............................. & 5,285 & 5,585 & 5,415 & 4,345 & 776 & 770 & 842 & 719 & & & & & & & & \\
\hline \multicolumn{17}{|l|}{ September 1996} \\
\hline Spartina patens ........ & 7,925 & 280 & 4,700 & 190 & 742 & 60 & 356 & 23 & $66(2)$ & $80(4)$ & $50(5)$ & $69(9)$ & ${ }^{3} 1.33$ & 31.64 & ${ }^{3} 1.06$ & ${ }^{3} 1.41$ \\
\hline Spartina alterniflora & 75 & 335 & 215 & 310 & 73 & 745 & 110 & 764 & -- & -- & $50(3)$ & ${ }^{4} 118(3)$ & - & -- & - & -- \\
\hline Distichlis spicata..... & 0 & 0 & 0 & 0 & 0 & 0 & 0 & 0 & -- & -- & -- & -- & -- & -- & -- & -- \\
\hline 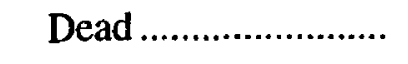 & -- & -- & -- & -- & 32 & 0 & 29 & 15 & -- & -- & $\cdots$ & -- & -- & -- & -- & -- \\
\hline Total ........................ & 8,000 & 615 & 4,915 & 500 & 847 & 805 & 495 & 802 & & & & & & & & \\
\hline
\end{tabular}



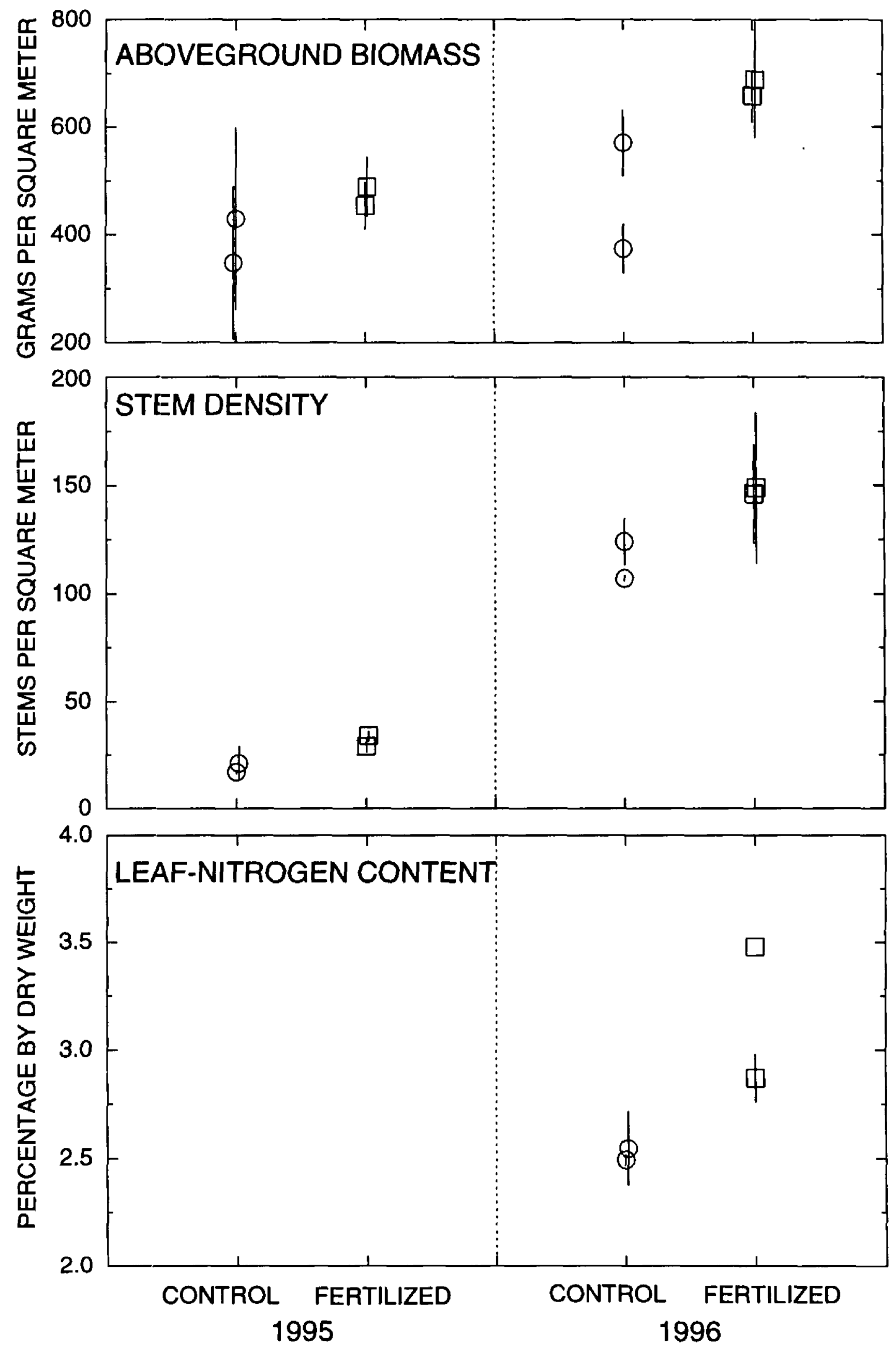

A.

Figure 7. Aboveground biomass, stem density, and leaf-nitrogen content in experimental (fertilized) and control plots, Inner Namskaket Marsh, Cape Cod, Massachusetts,

September 1995 and 1996. A. Phragmites. B. Spartina. 



I STANDARD DEVIATION $=+l-1$

O SPARTINA PATENS

$\triangle$ SPARTINA ALTERNIFLORA

B.

Figure 7. Aboveground biomass, stem density, and leaf-nitrogen content in experimental (fertilized) and control plots, Inner Namskaket Marsh, Cape Cod, Massachusetts, September 1995 and 1996-Continued. 


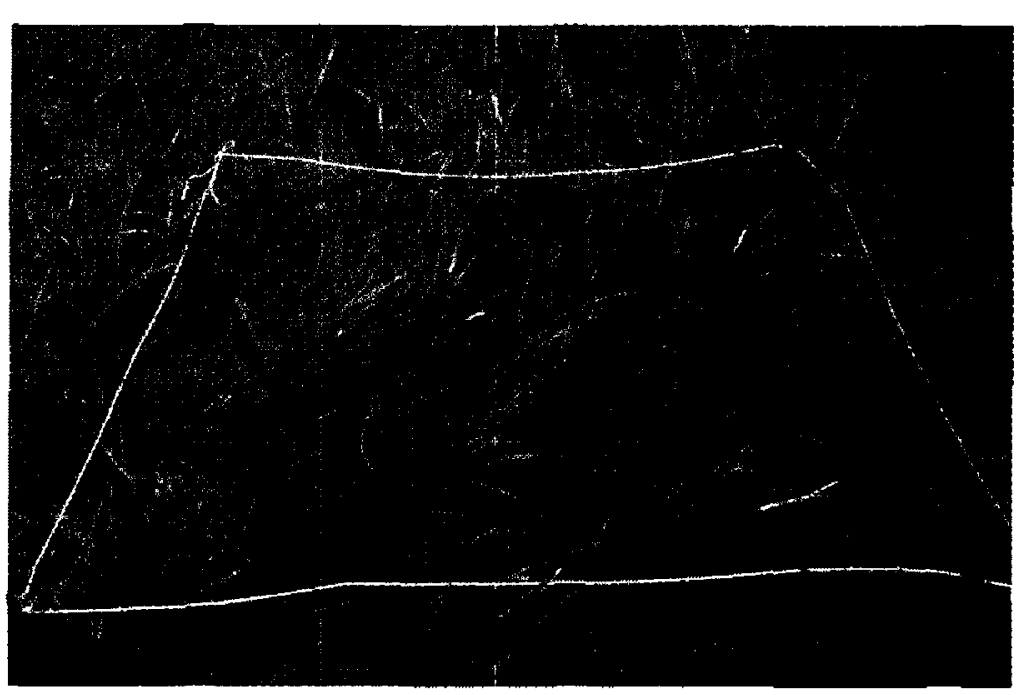

A.

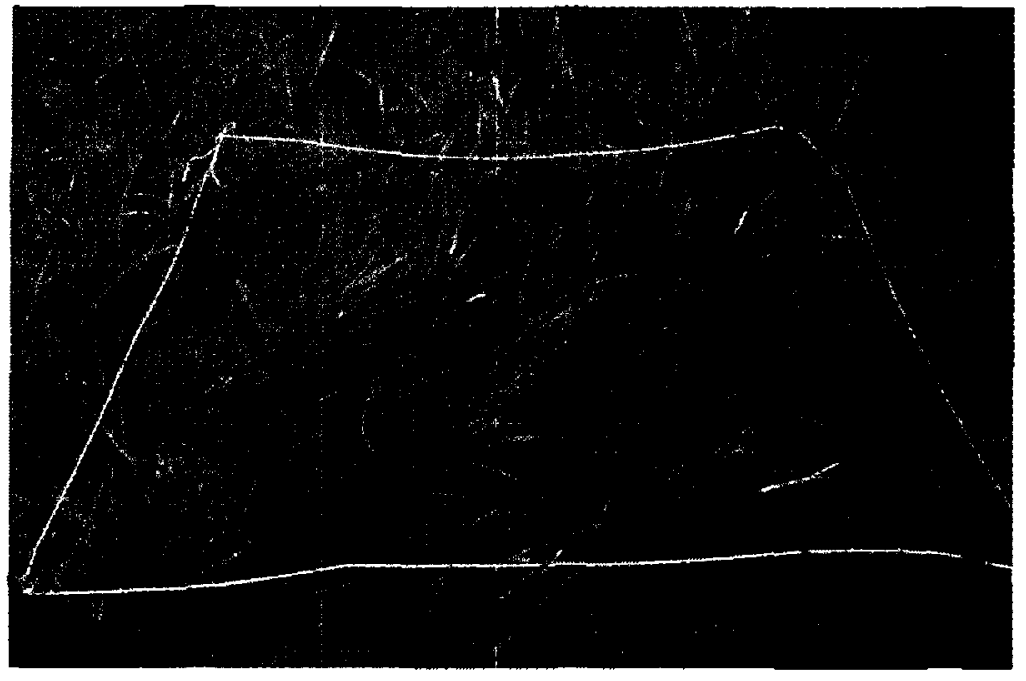

C.

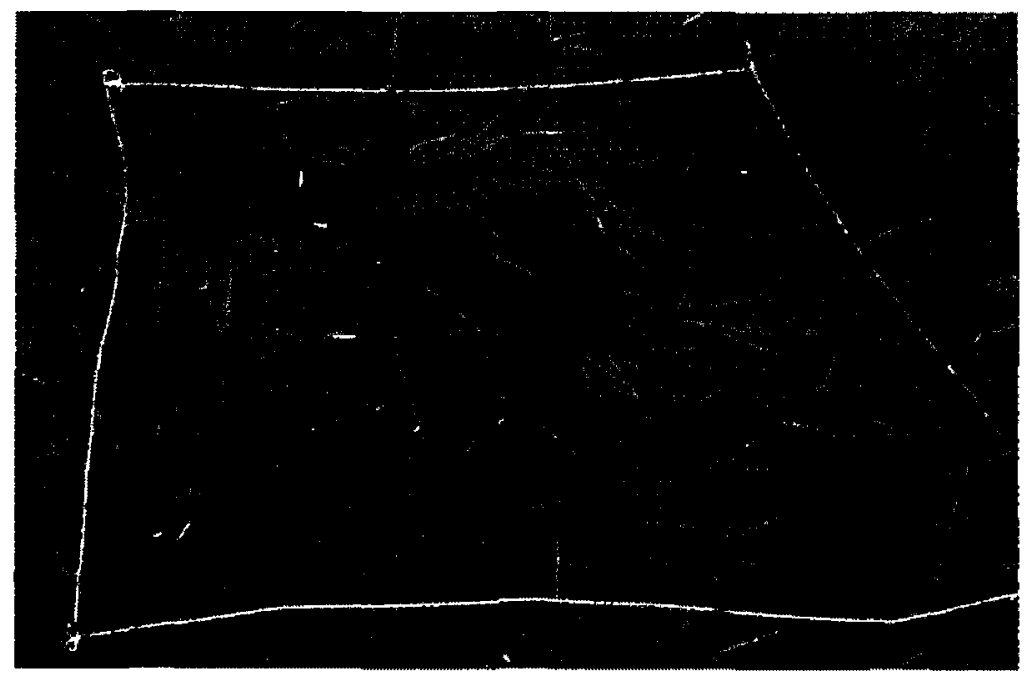

B.

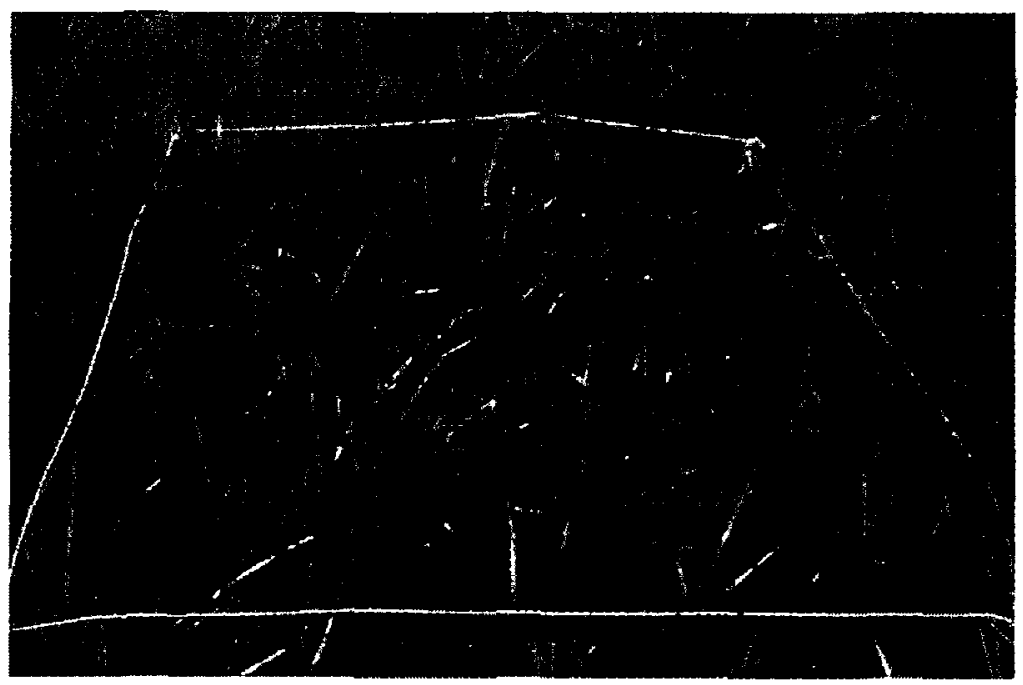

D.

Figure 8. Distribution of Spartina alterniflora and Spartina patens in nitrogen-enrichment experimental (C and D) and control (A and B) plots, Inner Namskaket Marsh, Cape Cod, Massachusetts, September 1995.

\section{ALGAE}

Macro- and micro-epibenthic algae are present on salt-marsh surfaces and in tidal creeks, although little is known about their community composition and dynamics (Nixon, 1986; Mitsch and Gosselink, 1993). In this study, data were collected to document the occurrence, distribution, and abundance of macro- and micro-epibenthic algae in the creek environment. The tidal creeks are the areas that would be most immediately affected by nitrogen loading from effluent-contaminated ground water, because ground water discharges primarily either through the boundary seepage zones, which are drained by the creek system, or directly through the creek bottoms (Howes and others,
1996). Microalgae (for example, green algae, bluegreen algae, and diatoms) also are present on the marsh surface under vegetation (especially $S$. alterniflora and Salicornia) and in pools and pannes. However, microalgae generally are not abundant in light-limited areas under densely spaced $S$. patens and Distichlis spicata stems, which cover much of Inner Namskaket Marsh (Blum, 1968, as reported in Nixon, 1982; Teal, 1986), and pools or pannes are not areally significant in the study area.

Macroalgae in the creek, qualitatively surveyed in late July/early August 1994, consisted mostly of Rhizoclonium and Bangia; a small area of Enteromorpha also was found just upstream of the 


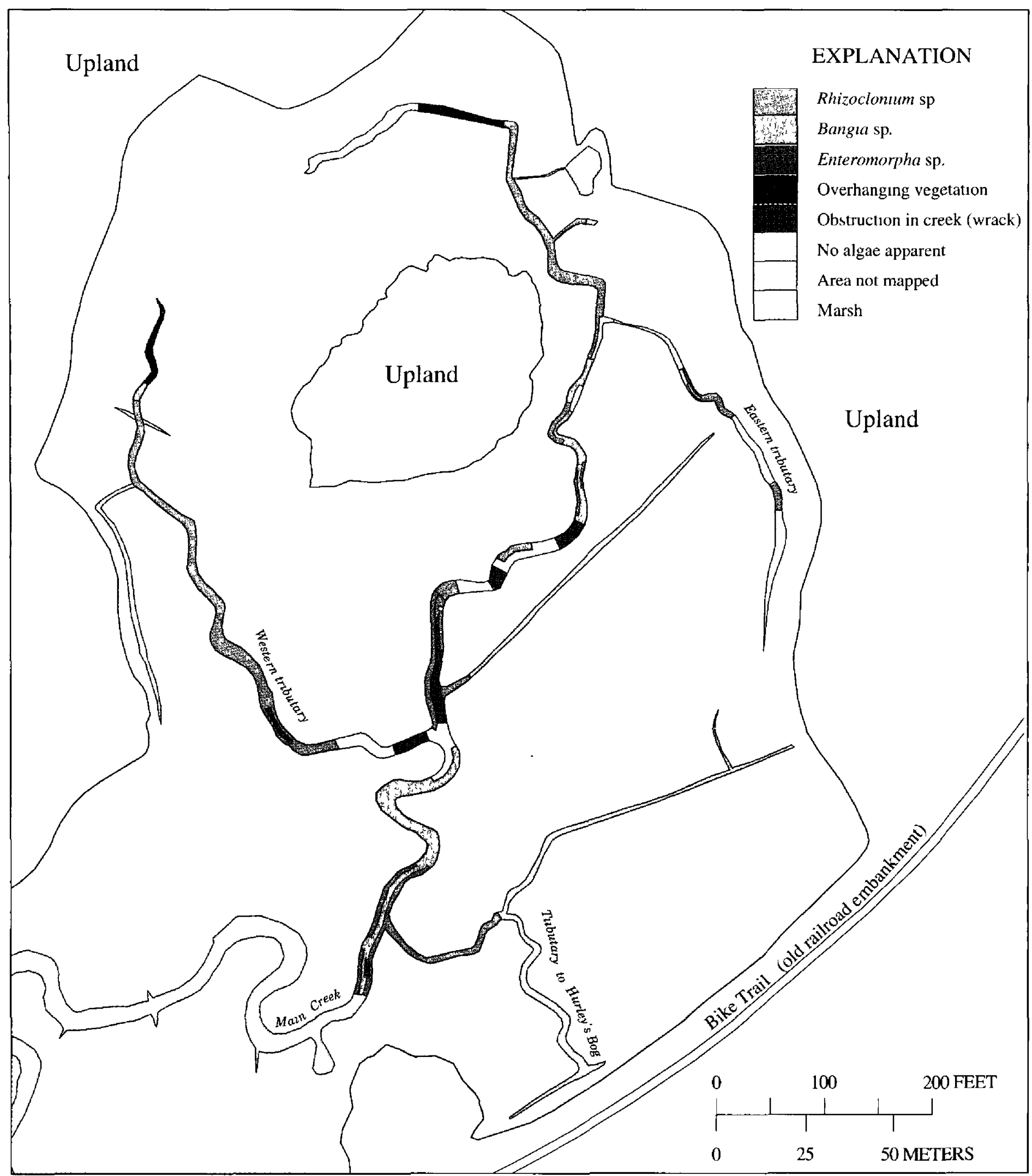

Figure 9. Areal distribution of macroalgae in creek channels of upper Namskaket Creek, Inner Namskaket Marsh, Cape Cod, Massachusetts, July 1994.

gaging station (figs. 3 and 9). Such low species diversity is typical of macroalgae communities in the upper reaches of salt-marsh environments (Nienhuis, 1994). Rhizoclonium is a very fine, filamentous green alga that grows annually in tangled masses and mats on peaty sediments, sand, or rocks, and Bangia is a filamentous red alga that grows annually or possibly perennially attached to hard substrates (such as wood or rocks) in intertidal environments (Taylor, 1957;

Kingsbury, 1969; Gosner, 1978; Villalard-Bohnsack and others, 1988). Rhizoclonium and Enteromorpha also were found with other genera in intertidal creek environments in nearby Nauset Marsh (Roman and others, 1996). In the upper reaches of the main creek 
and western tributary, overhanging vegetation (mostly Phragmites) appeared to prevent visible algal growth (fig. 9). Other areas of the creek were obstructed by wrack. Wrack deposits in the creek were dynamic, and areas where no algae was apparent during the 1994 survey may have been recently wrack covered. (The western tributary from T4 to T6 was filled with wrack during most of the 1995 and 1996 growing seasons.) Because of changing light and nutrient conditions, consumption by herbivorous grazers, disturbance by wrack and other floating material, and other factors, the distribution and biomass of algal species in the creek environments is variable and dynamic. Thus, the patterns shown in figure 9 represent a snapshot in time of a changing community.

Multiple surveys of the density and distribution of the epibenthic microalgal community in the marsh creeks were made using chlorophyll- $a$ concentrations in creek sediments. Chlorophyll- $a$ is a green pigment that is essential for photosynthesis and is a commonly used indicator of algal biomass. The dynamics of the microalgal community were monitored because microalgae are likely to be among the first organisms to show effects of increased nitrogen loading to a marsh; epibenthic microalgae grow year-round and are capable of more rapid production than macroalgae or emergent plants (Van Raalte and others, 1976). A detailed survey of chlorophyll- $a$ concentrations in the main creek was conducted in July 1995; marsh-wide surveys were conducted in April and May 1996 followed by biweekly to monthly sampling of seven sites throughout the growing season (table 6 and fig. 3)

Chlorophyll- $a$ concentrations were highly variable at individual sites $(A, B$, and $C$ samples, taken from the right, center, and left submerged creek bottoms at low tide), along creek channels, and across the marsh (table 6 and fig. 10A); the apparent higher variability in concentration with distance along the main creek channel in 1995 resulted from the more closely spaced sampling in that survey than in 1996. The high variability and patchy distribution is typical of chlorophyll- $a$ concentrations in dynamic environments like the marsh creek bottoms and results primarily from heterogeneity in flow patterns, grazing by invertebrates, and variable light conditions.

Concentrations across the marsh ranged from 0.2 to 95 $\mu \mathrm{g} / \mathrm{cm}^{2}$ in April and May 1996 and from 0.2 to $46 \mu \mathrm{g} / \mathrm{cm}^{2}$ in July 1995; the lower values are consistent with chlorophyll- $a$ concentrations in creek sediments measured at similar sites in Great Sippewissett Marsh in Falmouth, Cape Cod $\left(4.5 \mu \mathrm{g} / \mathrm{cm}^{2} \pm 0.22, n=4, \pm 1\right.$ SD; Wiltse and others, 1984). At several sites, concentrations appeared to peak in late May/early June, which may reflect changing light conditions, as overhanging vegetation increases during the summer, and(or) life-cycle characteristics of the individual algal species (fig. 10B; Kingsbury, 1969; Van Raalte and others, 1976). Chlorophyll- $a$ concentrations generally were higher in the upper reaches of the main creek and eastern tributary than in the western tributary or farther downstream in the main creek (table 6 and figs. 3 and $10 B$ ). The high chlorophyll- $a$ concentrations in the upper reaches of the main creek and eastern tributary may result from the high concentrations of dissolved inorganic nitrogen (nitrate, nitrite, and ammonium) in these waters. Dissolved inorganic nitrogen concentrations (April and May, 1994-95, $n=8$ ) averaged 0.52 and $0.92 \mathrm{mg} / \mathrm{L}$ as nitrogen in the eastern tributary and the upper reaches of the main creek, respectively, versus 0.10 and $0.43 \mathrm{mg} / \mathrm{L}$ as nitrogen in the western tributary and at the gaging station, respectively. The high chlorophyll- $a$ concentrations measured in the creek-bottom sediments represent productive microalgal populations. 
Table 6. Chlorophyll-a concentrations in creek-bottom sediments, Inner Namskaket Marsh, Cape Cod, Massachusetts, April through October 1996

[Site and creek: See figure 3 for location of sampling sites. E, eastern tributary; W, western tributary; M, main creek; looking downstream, samples A and B are from the right and left sides, respectively, of the submerged creek bottom at low tide, $C$ is from the creek-bottom center, and samples $D$ and $E$ are from 'right and left sides, respectively, of the emerged creek bottom/creek bank at low tide. Chlorophyll-a concentration: Where accompanied by number in parentheses, value shown is mean of two replicate samples and number in parentheses is standard deviation. BD, below detection limit; --, not sampled]

\begin{tabular}{|c|c|c|c|c|c|c|c|c|c|c|c|c|c|}
\hline \multirow{2}{*}{$\begin{array}{l}\text { Site } \\
\text { No. } \\
\text { and } \\
\text { creek }\end{array}$} & \multirow{2}{*}{ Sample } & \multicolumn{12}{|c|}{ Chlorophyll-a concentration, in mlcrograms per square centimeter } \\
\hline & & Apr. 12 & May 9 & May 23 & June 5 & June 18 & July 3 & July 23 & Aug. 2 & Aug. 15 & Aug. 21 & Aug. 29 & Oct. 13 \\
\hline \multirow[t]{3}{*}{$1 \mathrm{E}$} & A & 92 & 8.3 & - & 2.5 & 3.5 & 2.1 & 2.8 & 3.7 & -- & -- & -- & -- \\
\hline & B & 5.1 & 2.3 & -- & 4.2 & 3.0 & 3.0 & 3.2 & 4.9 & -- & -- & -- & -- \\
\hline & $\mathrm{C}$ & 5.6 & 1.2 & .. & 4.4 & 3.0 & 6.5 & 1.9 & 3.5 & -- & -- & -- & -- \\
\hline \multirow[t]{3}{*}{$2 \mathrm{E}$} & A & 20 & 3.0 & 44 & -- & -- & -- & -- & 16 & -- & -- & -- & -- \\
\hline & B & 2.8 & 3.7 & 9.7 & -- & -- & -- & -- & 28 & -- & -- & -- & -- \\
\hline & C & 3.0 & .7 & 5.1 & -- & -- & -- & -- & 9.5 & -- & -- & -- & -- \\
\hline $3 \mathrm{E}$ & A & 4.2 & 8.1 & -- & -- & -- & - & -- & - & -- & -. & -- & -- \\
\hline \multirow[t]{2}{*}{ 。 } & B & 7.9 & 5.6 & -- & -- & -- & -- & -- & -- & -- & - & -- & -- \\
\hline & $\mathrm{C}$ & 5.1 & 13 & -- & -- & -- & -- & -- & -- & -- & -- & -- & - \\
\hline \multirow[t]{3}{*}{$4 \mathrm{E}$} & A & 10 & 5.3 & -- & -- & - & -- & -- & -. & - & -- & -. & -- \\
\hline & B & 25 & 2.1 & -- & -- & -- & -- & - & -- & -- & -- & -- & - \\
\hline & $\mathrm{C}$ & 3.5 & 4.2 & -- & -- & -- & -- & -- & -- & -- & -- & -- & -- \\
\hline \multirow[t]{3}{*}{$5 \mathrm{E}$} & A & 1.9 & 6.7 & 12 & 2.5 & 4.2 & 7.9 & 5.8 & .9 & -- & -- & 15 & -- \\
\hline & B & 4.6 & 2.5 & 13 & 37 & 2.1 & 17 & 33 & 1.6 & -- & -- & 20 & -- \\
\hline & C & 4.9 & 1.2 & 16 & 2.5 & 20 & 5.1 & .2 & 17 & -- & -- & 20 & -- \\
\hline \multirow[t]{3}{*}{$6 \mathrm{E}$} & A & 4.6 & 3.9 & -- & 3.7 & -. & -- & -- & -- & -- & -- & -- & 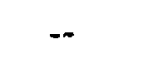 \\
\hline & B & 4.4 & 1.6 & -- & 1.9 & -. & -- & -- & -- & -- & -- & -- & -- \\
\hline & C & 12 & 6.7 & -- & 2.5 & - & - & -- & -- & -- & -- & -- & -- \\
\hline \multirow[t]{3}{*}{$7 \mathrm{~W}$} & $\mathbf{A}$ & 4.6 & 5.1 & -- & -- & -. & -- & -- & -- & -. & -- & -- & -- \\
\hline & B & 1.9 & 5.3 & -- & - & -- & -- & -- & - & -- & -- & -- & -- \\
\hline & $\mathrm{C}$ & 1.6 & 1.9 & -- & -- & -- & -- & -- & -- & -- & -- & -- & -- \\
\hline \multirow[t]{3}{*}{$8 W$} & A & .5 & .7 & - & -- & -- & -- & -- & -- & -- & -- & -- & -- \\
\hline & B & 3.2 & 1.9 & -- & -- & -- & -- & -- & -- & -- & -- & - & -- \\
\hline & $\mathrm{C}$ & .2 & .5 & -- & -- & -- & -- & .- & -- & -- & -- & -- & -- \\
\hline \multirow[t]{5}{*}{$9 \mathrm{~W}$} & A & -- & .2 & 1.4 & .2 & 2.1 & .7 & 29 & $7.9(3.8)$ & .7 & .2 & 12 & 0.2 \\
\hline & B & $\mathrm{BD}$ & 8.8 & $\mathrm{BD}$ & .7 & .2 & 19 & 7.9 & $12(4.4)$ & .9 & .5 & 20 & 2.3 \\
\hline & $\mathrm{C}$ & .2 & .5 & .7 & .7 & .5 & .5 & 13 & $5.4(3.1)$ & $\mathrm{BD}$ & 1.4 & 20 & 0.2 \\
\hline & D & -- & -- & -- & -- & -- & -- & 2.8 & -- & -- & -- & -- & -- \\
\hline & E & -- & -- & - & - & -- & -- & .5 & -- & -- & -- & -- & - \\
\hline \multirow[t]{3}{*}{$10 \mathrm{~W}$} & A & -- & .5 & -- & -- & -- & -- & -- & -- & -- & -- & -- & - \\
\hline & B & .5 & .5 & -- & -- & -- & -- & - & -- & -- & -- & -- & - \\
\hline & C & $\mathrm{BD}$ & .5 & -- & -- & -- & - & -- & -- & -- & -- & -- & -- \\
\hline \multirow[t]{3}{*}{$11 \mathrm{~W}$} & $\mathbf{A}$ & .5 & 1.4 & 3.9 & 7.2 & 3.9 & .9 & 3.0 & $2.8(1.0)$ & .9 & 5.3 & 4.6 & - \\
\hline & B & 3.5 & 10 & 14 & 1.9 & 3.5 & .7 & 3.9 & $1.7(.2)$ & 1.4 & .5 & 7.4 & -- \\
\hline & C & 7.6 & 3.0 & 74 & 3.7 & 16 & 8.3 & 3.2 & $5.5(3.3)$ & 1.2 & 4.2 & 17 & -- \\
\hline \multirow[t]{3}{*}{$12 \mathrm{~W}$} & A & 3.9 & 4.9 & -- & -- & -- & -- & -- & -- & -- & -- & -- & -- \\
\hline & B & 5.3 & 5.6 & -- & -- & -- & -- & -- & -- & -- & -- & -- & -- \\
\hline & $\mathrm{C}$ & 3.5 & 4.4 & -- & -- & -- & $\cdots$ & - & -- & -. & -- & $\cdots$ & - \\
\hline
\end{tabular}


Table 6. Chlorophyll-a concentrations in creek-bottom sediments, Inner Namskaket Marsh, Cape Cod, Massachusetts, April through October 1996-Continued

\begin{tabular}{|c|c|c|c|c|c|c|c|c|c|c|c|c|c|}
\hline \multirow{2}{*}{$\begin{array}{l}\text { Site } \\
\text { No. } \\
\text { and } \\
\text { creek }\end{array}$} & \multirow{2}{*}{ Sample } & \multicolumn{12}{|c|}{ Chlorophyll-a concentration, in micrograms per square centimeter } \\
\hline & & Apr. 12 & May 9 & May 23 & June 5 & June 18 & July 3 & July 23 & Aug. 2 & Aug. 15 & Aug. 21 & Aug. 29 & Oct. 13 \\
\hline \multirow[t]{3}{*}{$13 \mathrm{~W}$} & A & 0.2 & 2.5 & -- & -- & -- & -- & -. & -- & -- & -- & -- & -- \\
\hline & B & BD & 3.7 & -. & -- & -- & -- & -- & -- & - & -- & -- & -- \\
\hline & $\mathrm{C}$ & .2 & 8.6 & -- & -- & -- & -- & -- & -- & -- & -- & -- & -- \\
\hline \multirow[t]{3}{*}{$14 \mathrm{~W}$} & A & .7 & .5 & -- & -- & -- & -- & -- & -. & -- & -- & -- & -- \\
\hline & B & .5 & 2.1 & -- & -- & -. & -- & -- & -. & -- & -- & -- & -- \\
\hline & C & 13 & 2.5 & -- & -- & -- & -- &.- & -- & -- & -- & -- & -- \\
\hline \multirow[t]{3}{*}{$15 \mathrm{M}$} & A & -- & 6.9 & -- & -- & -- & -. & -- & -- & -- & -. & .- & -- \\
\hline & B & 2.3 & 14 & -- & -- & .- & -- & -- & .. & -- & -- & .- & .- \\
\hline & $\mathrm{C}$ & $\mathrm{BD}$ & 4.4 & -. & - & -- & .. & -- & .. & -- & .. & -- & - \\
\hline \multirow[t]{3}{*}{$16 \mathrm{M}$} & A & 21 & 16 & -- & -. & -- & -. & -- & -- & - & -- & -- & -- \\
\hline & B & 5.3 & .7 & -. & -- & -- & $\ldots$ & -- & --. & -- & -- & .. & .- \\
\hline & $\mathrm{C}$ & .7 & .5 & -- & - & -- & -. & -- & .. & -- & -. & -- & $s-$ \\
\hline \multirow[t]{3}{*}{$17 \mathrm{M}$} & A & .5 & 3.5 & -- & -. & -- & -- & -- & -- & - & -- & -- & -- \\
\hline & B & BD & 2.8 & -- & -- & -. & -- & -. & -- & - & -- & -- & -- \\
\hline & $\mathrm{C}$ & 4.4 & 9.0 & -- & - & -- & .. & -- & .. & -- & -- & -- & -- \\
\hline \multirow[t]{4}{*}{$18 \mathrm{M}$} & A & -- & 27 & 18 & 13 & BD & 1.4 & 42 & $23(17)$ & 0.2 & 0.7 & 23 & 0.9 \\
\hline & B & 2.5 & 9.7 & 9.3 & 8.8 & .9 & .2 & 29 & $2.9(.8)$ & .2 & 20 & 15 & 15 \\
\hline & C & 1.2 & 19 & .9 & 13 & 21 & .9 & 1.2 & $33(6.4)$ & 8.1 & .5 & 35 & .2 \\
\hline & D & -- & -. & -- & - & -- & -. & 10 & -- & -- & -- & -- & -- \\
\hline \multirow[t]{5}{*}{$19 \mathrm{M}$} & A & -- & 1.2 & BD & 60 & 134 & 5.8 & 6.7 & $24(11)$ & 3.5 & 2.8 & 2.1 & 1.4 \\
\hline & B & -- & 11 & .5 & 84 & 65 & 1.9 & 1.9 & $7.5(2.5)$ & 3.2 & 7.6 & 6.3 & .5 \\
\hline & C & 1.6 & 1.4 & 14 & 3.9 & 2.1 & 18 & 20 & $3.7(.7)$ & 25 & 29 & 12 & .5 \\
\hline & D & -- & -.. & -- & -- & -- & -- & 59 & -- & -- & -- & -- & -- \\
\hline & $\mathrm{E}$ & -- & -- & -- & -- & -- & -- & 31 & -. & -- &.- & -- & -- \\
\hline \multirow[t]{3}{*}{$20 \mathrm{M}$} & A & $\mathrm{BD}$ & .7 & -- & -- & -- & -- & - & $1.3(.5)$ & 29 & 6.3 & 10 & -- \\
\hline & B & -- & 15 & -- & -- & -- & -- & -- & $.4(.2)$ & 3.2 & 1.2 & 1.2 & -- \\
\hline & C & $\mathrm{BD}$ & 0 & -- & -. & -- & -. & -- & $20(22)$ & 5.3 & 1.4 & 28 & -- \\
\hline \multirow[t]{3}{*}{$21 \mathrm{M}$} & A & 6.9 & 8.1 & -- & -- & -- & -- & -- & $10(11)$ & .7 & 1.6 & 7.4 & 37 \\
\hline & B & 3.7 & 5.3 & -. & -. & -- & -- & -- & $14(1.8)$ & 6.0 & 9.0 & 24 & 13 \\
\hline & C & 5.6 & 4.6 & - & -- & -. & -. & - & $9.1(10)$ & 3.7 & 4.6 & 43 & -- \\
\hline \multirow[t]{3}{*}{$22 \mathrm{M}$} & A & 0.9 & 4.2 & -. & -- & .. & .. & -- & $6.2(6.2)$ & 0.2 & 14 & 38 & 8.1 \\
\hline & B & .7 & 1.4 & -- & -- & -- & -- & - & $5.6(1.0)$ & .5 & 1.2 & 1.4 & 4.2 \\
\hline & $\mathrm{C}$ & .9 & .5 & -- & -- & -- & -- & -. & $12(2.0)$ & .2 & 12 & 1.9 & 1.4 \\
\hline \multirow[t]{3}{*}{$23 \mathrm{M}$} & A & .7 & 3.9 & -- & -- & -- & -- & .. & $4.4(4.2)$ & .2 & 3.7 & 108 & .7 \\
\hline & B & 1.4 & 2.1 & -- & -. & -. & -. & -- & $8.4(10)$ & 11 & 49 & 31 & 35 \\
\hline & C & 1.9 & 72 & -. & -- & -- & -- & -- & $22(3.1)$ & 2.3 & 28 & 17 & .5 \\
\hline \multirow[t]{3}{*}{$24 M$} & A & 1.2 & 22 & 20 & 37 & 3.0 & 69 & 34 & $1.4(2.0)$ & BD & 1.4 & 66 & 2.8 \\
\hline & B & 3.2 & 2.8 & 140 & 170 & 10 & 38 & 43 & $14(13)$ & 6.7 & 4.9 & 1.2 & .9 \\
\hline & C & 8.1 & .7 & 1.6 & 130 & 5.1 & 8.1 & 15 & $.9(.7)$ & .9 & .7 & 3.0 & .5 \\
\hline \multirow[t]{3}{*}{$25 \mathrm{M}$} & A & .5 & 42 & -- & -- & -- & -- & -. & -. & -. & -- & -- & .. \\
\hline & B & 10 & 95 & .- & -- & -- & -- & -- & .. & .. & -. & -- & .. \\
\hline & $\mathrm{C}$ & 1.6 & 23 & -- & -- & -- & -- & -- & -- & -- & -- & -- & -- \\
\hline
\end{tabular}




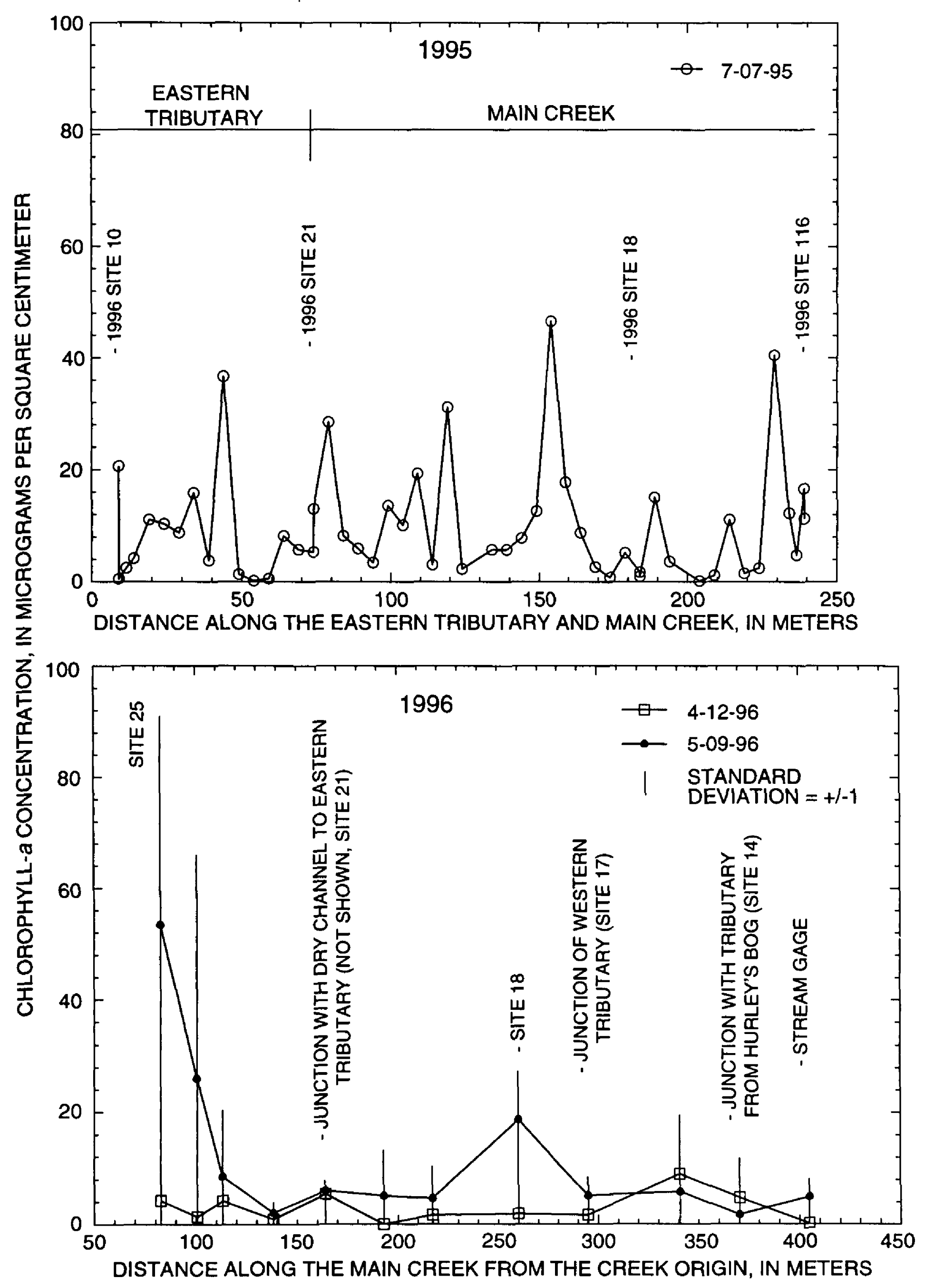

A.

Figure 10. Concentrations of chlorophyll-a in creek-bottom sediments, Inner Namskaket Marsh, Cape Cod, Massachusetts. A. July 1995 ( $n=1$ per site) and April and May 1996 ( $n=3$ per site). B. April through October 1996. 

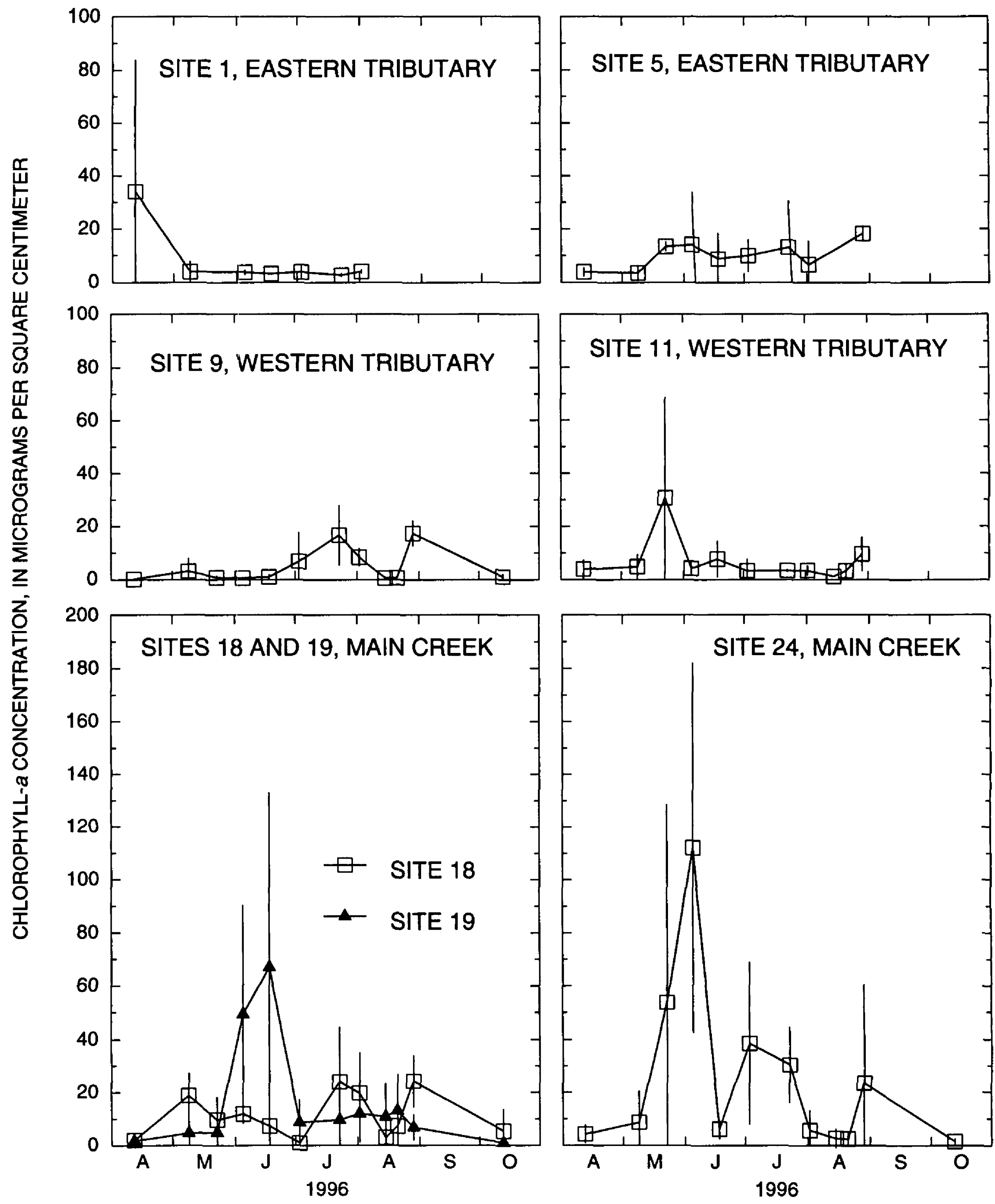

B.

Figure 10. Concentrations of chlorophyll-a in creek-bottom sediments, Inner Namskaket Marsh, Cape Cod, MassachusettsContinued. 


\section{SUMMARY AND CONCLUSIONS}

Namskaket Marsh is an 80-hectare salt marsh on the northeastern coast of Cape Cod, Massachusetts. Inland parts of the marsh are adjacent to a septagetreatment facility and are possible discharge areas for ground water contaminated by nitrogen-rich effluent from the facility. Nitrogen is the primary limiting nutrient to salt marsh plants and algae. Thus, increased nitrogen loading to Namskaket Marsh through discharge of nitrogen-enriched ground water from the treatment facility or from other sources has the potential to alter the distribution and(or) productivity of wetland plants and algae and indirectly affect other wildlife in the marsh. The study area consists of a 7hectare inland lobe of Namskaket Marsh (Inner Namskaket Marsh) and an adjacent 1.3-hectare brackish marsh, Hurley's Bog. The study, conducted in 1994-96 as part of a larger investigation of the effluent plume and hydrology and nitrogen-remediation potential of the marsh, focused on those areas and characteristics of the marsh that most likely would experience or be affected by increased nitrogen loading from discharge of the effluent-contaminated ground water.

Inner Namskaket Marsh is a typical New England high salt marsh with brackish-water transition zones along the marsh/upland border. It is drained by tidal creeks and most of its study area is flooded only by the bimonthly spring tides and less frequent storm tides. The marsh supports a diverse flora of flowering plants, distributed in a mosaic-like pattern. Pure stands and mixed assemblages of Phragmites australis (common reed), Spartina patens (salt-marsh hay), Spartina alterniflora (cordgrass), and Distichlis spicata (spike grass) cover most (73 percent) of the mapped area. Phragmites australis occurs primarily in irregular bands along the marsh/upland boundary, in areas of low pore-water salinity (4 to $8 \mathrm{ppt}$ and less) that are influenced by ground-water seepage. Other plants tolerant of brackish water, Scirpus robustus (robust bulrush), Iva frutescens (marsh elder), Spartina cynosuroides (big cordgrass), and Typha sp. (cattail) also occur near marsh/upland boundaries. Spartina alterniflora (tall form) occurs along creekbanks of the main channel, which are frequently flooded, whereas Spartina patens and Distichlis spicata occur in patches primarily inland from creekbanks. Salicornia europaea (common glasswort) a colonizer of bare patches, grows in an irregular area that probably was recently covered by wrack (dead plant material). Wrack deposits covered about 15 percent of the mapped area in 1995 and appeared to be a significant factor affecting plant distribution. Other plants occurring in the marsh included Solidago sempervirens (seaside goldenrod) and Pluchea purpurascens (salt-marsh fleabane); Juncus gerardi (black grass), commonly present along upland borders in New England high marshes, is much less common than other brackish wetland plants in Inner Namskaket Marsh.

Hurley's Bog is covered primarily by Phragmites australis (about 80 percent of the open, herbaceous area); Iva frutescens, Scirpus robustus, Solidago sempervirens, and $S$. patens also are present. The open area is surrounded by a shrub oak/maple wet woodland. The distribution of herbaceous and woody plants in Hurley's Bog likely has been affected by the 19th-century construction of the railroad embankment that restricts tidal flow and separates the bog from Namskaket Marsh.

Aboveground biomass and stem density by species and leaf-nitrogen content were measured because they are quantitative measures of the existing plant community that could change with increased nitrogen loading to the marsh. Aboveground biomass, an indicator of annual production, ranged from 390 to $1,050 \mathrm{~g}$ of dry weight per $\mathrm{m}^{2}$ for various mixed associations of primarily Spartina patens, Spartina alterniflora, and Distichlis spicata. Leaf-nitrogen content, sampled in plant associations along the eastern marsh/upland border where ground-water seepage is greatest, averaged 3.18 $\pm 0.30( \pm 1$ standard deviation, $n=11)$ and $1.87 \pm 0.15( \pm 1$ standard deviation, $n=4)$ percent of dry weight in Phragmites australis and Scirpus robustus samples, respectively. These values are similar to reported values from other New England high marshes.

Results of a small-scale experiment showed that nitrogen addition, at a rate that approximated maximum estimated loading rates associated with discharge of the effluent-contaminated ground water in the marsh, affected plant growth in Phragmites australis and Spartina patens/ Spartina alterniflora associations. Aboveground biomass and leaf-nitrogen content of Phragmites increased under nitrogen addition. In the Spartina patens/Spartina alterniflora associations, nitrogen addition resulted in a species shift from Spartina patens to Spartina alterniflora rather than in an increase in aboveground biomass during the two growing seasons of the experiment. This species shift, if it occurred, could potentially increase the area of estuarine-nursery habitat in Inner Namskaket Marsh. 
Macro- and microalgal communities were surveyed in the tidal creeks in Inner Namskaket Marsh, because these environments likely would be the areas most immediately affected by nitrogen loading from effluent-contaminated ground water. In particular, changes in the epibenthic microalgae would likely be the first observable effect on plants of increased nitrogen loading to the marsh because epibenthic microalgae grow year-round and are capable of more rapid production than macroalgae or emergent plants. Macroalgae, surveyed in late July/early August 1994, consisted mostly of Rhizoclonium, a very fine, green filamentous alga that grows in tangled masses and mats on peaty sediments and other surfaces, and Bangia, a filamentous red alga that grows attached to hard substrates in intertidal environments. Wrack in the creek and overhanging vegetation appeared to affect the distribution of macroalgae in the creek.

Microalgae were surveyed by measuring concentrations of chlorophyll- $a$, a green pigment necessary for photosynthesis, in creek sediments. Marshwide surveys showed that chlorophyll- $a$ concentrations were highly variable at several scales of measurement and ranged between 0.2 and $95 \mu \mathrm{g} / \mathrm{cm}^{2}$ in April and May 1996. Concentrations appeared to peak at several sites in late May/early June, which may reflect changing light conditions and(or) life-cycle characteristics of the individual algal species. Chlorophyll- $a$ concentrations generally were higher in the upper reaches of the main creek and eastern tributary than in the western tributary or farther downstream in the main creek. The high chlorophyll-a concentrations in the former areas may result from the relatively high concentrations of dissolved inorganic nitrogen in these waters $(0.52$ and $0.92 \mathrm{mg} / \mathrm{L}$ as nitrogen in the eastern tributary and the upper reaches of the main creek, respectively, average April/May concentrations). The high chlorophyll- $a$ concentrations measured in the creek-bottom sediments represent productive microalgal populations. The effect of increasing nitrogen concentrations in the creeks is likely to be small, because creek nitrogen concentrations are already high relative to the nitrogenuptake rates of the populations. Results of a nitrateaddition experiment in the creeks (August through October, 1996) did not suggest an ecologically significant increase in chlorophyll-a concentrations or macroalgae during the summer months.
Inner Namskaket Marsh is a dynamic and diverse wetland environment that is subject to natural and man-made disturbances. Increased nitrogen loading from discharge of nitrogen-enriched ground water originating at the nearby treatment facility or other sources could potentially alter the existing plant and algal communities. Results of this study suggest that the expected responses of the dominant species in the marsh initially would be a modest increase in aboveground production and leaf-nitrogen content of Phragmites and a potential species shift in Spartina patens/ Spartina alterniflora assemblages to Spartina alterniflora in affected areas. However, the effects of increased nitrogen loading on Spartina patens/ Spartina alterniflora assemblages are unlikely to be widespread, because ground- and surface-water transport of plume nutrients to interior areas of the marsh (where Spartina patens is dominant) is likely to be limited. In addition, the present-day, small-scale distribution of plant associations in Inner Namskaket marsh will continue to be affected by wrack. Wrack deposition and removal is a natural feature of the marsh and affects the distribution and abundance of Spartina patens, Distichlis spicata, Salicornia, and other species. However, although the size and distribution of specific patches of plants may vary from year to year due to wrack movements, the presence of the plant species and associations is likely to be relatively stable over time in the absence of other changes.

For Namskaket Marsh, leaf-nitrogen content of the Phragmites in the brackish transition zone along the marsh/upland boundary, creek-water nitrogen concentrations, and chlorophyll- $a$ concentrations in creek sediments would likely be the best indicators of increased nitrogen loading to the marsh from discharge of the septage-effluent plume or other ground-water sources. Long-term monitoring of species distribution also would indicate significant changes in marsh ecology from changes in nitrogen loading or hydrology. These indicators would work best for Namskaket Marsh because of the extensive Phragmites-dominated brackish transition zone between the salt-marsh and upland areas. In salt marshes where halophytes are the dominant species up to the upland border, aboveground biomass and stem density, in addition to leaf-nitrogen content and the other indicators listed above, also would be indicators of changes in nitrogen loading. 


\section{REFERENCES CITED}

Bertness, M.D., 1992, The ecology of a New England salt marsh: American Scientist, v. 80, p. 260-268.

Bertness, M.D., and Ellison, A.M., 1987, Determinants of pattern in a New England salt marsh community: Ecological Monographs, v. 57, p. 129-147.

Bertness, M.D., Gough, L., and Shumway, S.W., 1992, Salt tolerances and the distribution of fugitive salt marsh plants: Ecology, v. 73, p. 1842-1851.

Dacey, J.W.H., and Howes, B.L., 1984, Water uptake by roots controls water table movement and sediment oxidation in short Spartina marsh: Science, v. 224, p. 487489.

DeSimone, L.A., Barlow, P.M., and Howes, B.L., 1996, A nitrogen-rich septage-effluent plume in a glacial aquifer, Cape Cod, Massachusetts, February 1990 through December 1992: U.S. Geological Survey Water-Supply Paper 2456, $89 \mathrm{p}$.

DeSimone, L.A., and Howes, B.L., 1995, Hydrogeologic, water-quality, and biogeochemical data collected at a septage-treatment facility, Orleans, Cape Cod, Massachusetts, October 1988 through December 1992: U.S. Geological Survey Open-File Report 95-439.

DeSimone, L.A., and Howes, B.L., 1996, Denitrification and nitrogen transport in a coastal aquifer receiving wastewater discharge: Environmental Science and Technology, v. 30, p. 1152-1162.

DeSimone, L.A., Howes, B.L., and Barlow, P.M., 1997, Mass-balance analysis of reactive transport and cation exchange in a plume of wastewater-contaminated groundwater: Journal of Hydrology, in press.

Ellison, A.M., 1987, Effects of competition, disturbance, and herbivory on Salicomia europaea: Ecology, v. 68, p. 576-586.

Frey, R.W., and Basan, P.B., 1985, Coastal salt marshes, in Davis, R.A., ed., Coastal Sedimentary Environments, 2nd edition: New York, Springer-Verlag, p. 225-301.

Giblin, A.E., and Gaines, A.G., 1990, Nitrogen inputs to a marine embayment: the importance of groundwater: Biogeochemistry, v. 10, p. 309-328.

Good, R.E., Good, N.F., and Frasco, B.R., 1982, A review of primary production and decomposition dynamics of the belowground marsh component, in Kennedy, V.S., editor, Estuarine Comparisons: New York, Academic Press, p. 139-157.

Gosner, K.L., 1978, A field guide to the Atlantic Seashore: Boston, Mass., Houghton Mifflin Company, 329 p.

Hartman, J.M., 1988, Recolonization of small disturbance patches in a New England salt marsh: American Journal of Botany, v. 75, p. 1625-1631.
Haslan, S.M., 1969, The development and emergence of buds in Phragmites communis Trin.: Annals of Botany, v. 33, p. 289-301.

Howes, B.L., Weiskel, P.D., Goehringer, D.D., and Teal, J.M., 1996, Interception of freshwater and nitrogen transport from uplands to coastal waters: the role of salt marshes, in Nordstrom, K.F., and Roman, C.T., eds, Estuarine Shores: Evolution, Environments and Human Alterations: London, John Wiley \& Sons, p. 287-310.

Kingsbury, J.M., 1969, Seaweeds of Cape Cod and the Islands: Chatham, Mass., Chatham Press, 212 p.

Linthurst, R.A., and Reimold, R.J., 1978, An evaluation of methods for estimating the net aerial primary production of estuarine angiosperms: Journal of Applied Ecology, v. 15, p. 919-931.

Miller, W.B., and Egler, F.E., 1950, Vegetation of the Wequetequock-Pawcatuck tidal marshes, Connecticut: Ecological Monographs, v. 20, p. 143-172.

Mitsch, W.J., and Gosselink, J.G., 1993, Wetlands, 2nd edition: New York, Van Nostrand Reinhold, $722 \mathrm{p}$.

Niering, W.A., and Warren, R.S., 1980, Vegetation patterns and processes in New England salt marshes: BioScience, v. 30, p. 301-307.

Nienhuis, P.H., 1994, Seaweeds in estuaries and salt marshes, in Lobban, C.S., and Harrison, P.J., Seaweed ecology and physiology: Cambridge, England, Cambridge University Press, p. 85-87.

Nixon, S.W., 1982, The Ecology of New England High Salt Marshes: A Community Profile: Washington, D.C., U.S. Fish and Wildlife Service, Biological Services Program, Report No. FWS/OBS-81/55, 70 p.

Oldale, R.N., Koteff, C., and Hartshorn, J.H., 1971, Geologic map of the Orleans Quadrangle, Barnstable County, Cape Cod, Massachusetts: U.S. Geological Survey Map GQ-931, 1 sheet.

Parsons, T.R., Maita, Y., and Lalli, C., 1989, Manual of chemical and biological methods for seawater analysis: Pergamon Press, $173 \mathrm{p}$.

Redfield, A.C., 1972, Development of a New England salt marsh: Ecological Monographs, v. 42, p. 201-237.

Roman, C.T., Able, K.W., Lazzari, M.A., and Heck, K.L., 1990 , Primary productivity of angiosperm and macroalgae dominated habitats in a New England salt marsh: a comparative analysis: Estuarine, Coastal, and Shelf Science, v. 30, p. 35-45.

Roman, C.T., and Daiber, F.C., 1984, Aboveground and belowground primary production dynamics of two Delaware Bay tidal marshes: Bulletin of the Torrey Botanical Club, v. 111, p. 34-41. 
Shisler, J.K., Shulze, T.L., and Howes, B.L., 1978, The effect of the marsh elder (Iva frutescens) on the standing crop biomass of Spartina patens and associated wildlife: Biological Conservation, v. 14, p. 159-166.

Smart, R.M., and Barko, J.W., 1980, Nitrogen nutrition and salinity tolerance of Distichlis spicata and Spartina alterniflora: Ecology, v. 61, p. 630-638.

Taylor, W.R., 1957, Marine Algae of the Northeastern Coast: Ann Arbor, Mich., University of Michigan Press, 509 p.

Teal, J.M., 1986, The ecology of regularly flooded salt marshes of New England: A community profile: Washington, D.C., U.S. Fish and Wildlife Service, Biological Report 87(7.4), $61 \mathrm{p}$.

Teal, J.M., and Howes, B.L., 1996, Interannual variability of a salt-marsh ecosystem: Limnology and Oceanography: v. 41, p. $802-809$.

Teal, J.M., and Giblin, A.E., 1983, The potential impact of the proposed disposal area on Namskaket Marsh, in Teal, J.M., The coastal impact of ground water discharge: an assessment of anthropogenic nitrogen loading in Town Cove, Orleans, Massachusetts: Woods Hole, Mass., Woods Hole Oceanographic Institution Final Report for Proposal No. 2778, Part 3, p. 149-159.

Tiner, R.W., Jr., 1987, A field guide to the coastal wetland plants of the northeastern United States: Amherst, Mass., The University of Massachusetts Press, 285 p.

Valiela, I., Costa, J., Foreman, K., Teal, J.M., Howes, B., and Aubrey, D., 1990, Transport of groundwater-borne nutrients from watersheds and their effects on coastal waters: Biogeochemistry, v. 10, p. 177-197.

Valiela, I., Teal, J.M., and Persson, N.Y., 1976, Production and dynamics of experimentally enriched salt marsh vegetation: belowground biomass: Limnology and Oceanography, v. 21, p. 245-252.

Valiela, I., and Teal, J.M., 1979, The nitrogen budget of a salt marsh ecosystem: Nature, v. 280, p. 652-656.
Valiela, I., Teal, J.M., Cogswell, C., Hartman, J., and Allen, S., 1985, Some long-term consequences in wetlands treatment of municipal wastewaters, in Farrington, J.W., ed., Ecological Considerations in Wetlands Treatment of Municipal Wastewaters: New York, Van Nostrand Reinhold Company, p. 301-316.

Van Raalte, C.D, Valiela, I., and Teal, J.M., 1976, Production of epibenthic salt marsh algae: light and nutrient limitations: Limnology and Oceanography, v. 21, p. 862-872.

Villalard-Bohnsack, Martine, Peckol, Paulette, and Harlin, M.M., 1988, Marine macroalgae of Narragansett Bay and adjacent sounds, in Sheath, R.G., and Harlin, M.M., eds., Freshwater and Marine Plants of Rhode Island: Dubuque, Iowa, Kendall/Hunt Publishing Company, ch. 12, p. 101-118.

Vince, S.W., Valiela, I., and Teal, J.M., 1981, An experimental study of the structure of herbaceous insect communities in a salt marsh: Ecology, v. 62, p. 1661-1678.

Weiskel, P.W., DeSimone, L.A., and Howes, B.L., 1996, A nitrogen-rich septage-effluent plume in a coastal aquifer, marsh, and creek system, Orleans, Massachusetts: project summary, 1988-95: U.S. Geological Survey Open-File Report 96-111, 20 p.

Weiskel, P.W., DeSimone, L.A., and Howes, B.L., 1997, The Namskaket Marsh project: nitrogen transport and ecosystem characterization in a Cape Cod aquifer and salt marsh: Environment Cape Cod, v. 1, no. 2, p. 10-27.

Weiskel, P.W., and Howes, B.L., 1992, Differential transport of sewage-derived nitrogen and phosphorus through a coastal watershed: Environmental Science and Technology, v. 26, p. 352-360.

Wiltse, W.I., Foreman, K.H., Teal, J.M., and Valiela, I., 1984, Effects of predators and food resources on the macrobenthos of salt marsh creeks: Journal of Marine Research, v. 42, p: 923-942. 
Table 7. Locations of vegetation and pore-water sampling sites along transects used to measure relative percentage of cover, stem density, and aboveground biomass, Inner Namskaket Marsh, Cape Cod, Massachusetts

[Location, in feet, in Massachusetts state-plane coordinates (zone 4151); ND, not determined]

\begin{tabular}{|c|c|c|c|c|c|}
\hline \multirow{2}{*}{ Sampling site } & \multicolumn{2}{|c|}{ Location } & \multirow{2}{*}{ Sampling site } & \multicolumn{2}{|c|}{ Location } \\
\hline & North & East & & North & East \\
\hline $\mathrm{T} 1-25$ & $288,509.2$ & $1,006,676.9$ & T6-600 & $288,933.4$ & $1,006,063.6$ \\
\hline $\mathrm{T} 2-10$ & $288,567.0$ & $1,006,731.1$ & T6-665 & $288,927.6$ & $1,005,999.6$ \\
\hline$T 2-60$ & $288,564.9$ & $1,006,680.6$ & $77-5$ & $289,064.4$ & $1,006,613.1$ \\
\hline$T 2-160$ & $288,558.9$ & $1,006,580.6$ & $\mathrm{~T} 7-50$ & $289,061,0$ & $1,006,570.1$ \\
\hline$T 2-260$ & $288,552.4$ & I, $006,481.5$ & $T 7-130$ & ND & ND \\
\hline T2-360 & $288,549.3$ & $1,006,381.1$ & $T 7-160$ & $289,053.9$ & $1,006,460.5$ \\
\hline$T 2-460$ & $288,543.8$ & $1,006,279.6$ & $\mathrm{~T} 7-410$ & ND & ND \\
\hline$T 2-560$ & $288,539.1$ & $1,006,180.7$ & $\mathrm{~T} 7-480$ & $289,040.3$ & $1,006,177.8$ \\
\hline$T 2-660$ & $288,533.6$ & $1,006,080.0$ & $\mathrm{~T} 7-550$ & $289,043.7$ & $1,006,108.0$ \\
\hline $\mathrm{T} 2-705$ & $288,531.0$ & $1,006,034.4$ & $T 7-610$ & $289,044.6$ & $1,006,048.6$ \\
\hline $\mathrm{T} 2-750$ & ND & ND & $\mathrm{T} 8.5$ & $289,128.8$ & $1,006,542.6$ \\
\hline T3-5 & $288,671.2$ & $1,006,683.4$ & $\mathrm{~T} 8-70$ & $289,129.8$ & $1,006,479.4$ \\
\hline $\mathrm{T} 3-50$ & $288,664.8$ & $1,006,639.4$ & T8-140 & $289,132.1$ & $1,006,411.1$ \\
\hline T3-100 & $288,658.6$ & $1,006,589.0$ & T8-210 & $289,126.5$ & $1,006,345.1$ \\
\hline T3-170 & $288,656.0$ & $1,006,520.7$ & T8-280 & $289,128.9$ & $1,006,273.8$ \\
\hline T3-240 & $288,651.9$ & $1,006,451.5$ & $\mathrm{~T} 8-350$ & $289,128.0$ & $1,006,202.2$ \\
\hline T3-310 & $288,650.9$ & $1,006,381.1$ & T8-420 & $289,135.9$ & $1,006,125.1$ \\
\hline T3-380 & $288,621.4$ & $1,006,311.0$ & T8-490 & $289,140.8$ & $1,006,066.7$ \\
\hline T3-450 & $288,623.8$ & $1,006,239.6$ & T9-15 & $289,222.6$ & $1,006,451.8$ \\
\hline T3-520 & $288,627.9$ & $1,006,171.0$ & T9-35 & $289,221.3$ & $1,006,433.0$ \\
\hline T3-570 & $288,633.6$ & $1,006,118.9$ & T9-115 & $289,215.2$ & $1,006,352.9$ \\
\hline T3-595 & $288,637.4$ & $1,006,094.0$ & T9-185 & $289,212.6$ & $1,006,280.7$ \\
\hline $\mathrm{T} 4-5$ & $288,770.4$ & $1,006,678.8$ & T9-255 & $289,207.8$ & $1,006,212.0$ \\
\hline $\mathrm{T} 4-50$ & $288,766.7$ & $1,006,635.1$ & T9-325 & $289,202.3$ & $1,006,141.9$ \\
\hline T4-120 & $288,763.2$ & $1,006,565.2$ & T9-395 & $289,197.7$ & $1,006,073.5$ \\
\hline T4-190 & $288,759.8$ & $1,006,495.4$ & T10-25 & $288,438.3$ & $1,006,607.5$ \\
\hline T4-260 & $288,756.2$ & $1,006,425.7$ & T10-100 & $288,499.1$ & $1,006,565.7$ \\
\hline T4-330 & $288,752.9$ & $1,006,366.2$ & T10-150 & $288,547.9$ & $1,006,532.6$ \\
\hline T4-400 & $288,747.6$ & $1,006,286.2$ & T11-25 & $288,376.3$ & $1,006,525.3$ \\
\hline $\mathrm{T} 4-470$ & $288,743.8$ & $1,006,216.0$ & T11-100 & $288,436.9$ & $1,006,483.3$ \\
\hline T4-573 & $288,737.9$ & $1,006,114.9$ & $\mathrm{~T} 11-175$ & $288,499.1$ & $1,006,443.1$ \\
\hline T4-618 & $288,731.7$ & $1,006,070.4$ & $\mathrm{~T} 12-25$ & $288,321.1$ & $1,006,438.5$ \\
\hline T5-0 & $288,895.4$ & $1,006,693.5$ & T12-100 & $288,381.4$ & $1,006,395.1$ \\
\hline T5-7 & $288,895.1$ & $1,006,687.0$ & T12-165 & $288,433.1$ & $1,006,357.8$ \\
\hline T5-13 & $288,892.6$ & $1,006,681.0$ & $\mathrm{~T} 12-240$ & $288,494.3$ & $1,006,317.2$ \\
\hline T5-30 & $288,886.6$ & $1,006,666.0$ & T13-50 & $288,395.1$ & $1,006,300.7$ \\
\hline T5-45 & $288,880.8$ & $1,006,650.7$ & T13-125 & $288,455.7$ & $1,006,259.4$ \\
\hline T5-82 & $288,869.4$ & $1,006,618.5$ & T13-200 & $288,518.3$ & $1,006,219.2$ \\
\hline T5-135 & $288,862.2$ & $1,006,563.5$ & T14-0 & $288,245.2$ & $1,006,248.3$ \\
\hline T5-203 & $288,852.5$ & $1,006,494.9$ & T14-25 & $288,265.0$ & $1,006,234.5$ \\
\hline T5-270 & $288,855.6$ & $1,006,430.4$ & T14-100 & $288,325.5$ & $1,006,191.6$ \\
\hline T5-277 & $288,853.6$ & $1,006,471.1$ & T14-175 & $288,389.5$ & $1,006,145.3$ \\
\hline T5-340 & $288,849.9$ & $1,006,360.5$ & T14-275 & $288,474.1$ & $1,006,084.9$ \\
\hline T5-410 & $288,844.6$ & $1,006,291.2$ & T15-25 & $288,194.9$ & $1,006,166.9$ \\
\hline T5-480 & $288,840.6$ & $1,006,221.3$ & $\mathrm{~T} 15-100$ & $288,254.7$ & $1,006,123.4$ \\
\hline T5-550 & $288,835.7$ & $1,006,150.8$ & T15-175 & $288,314.7$ & $1,006,080.5$ \\
\hline T5-620 & $288,832.8$ & $1,006,080.8$ & $\mathrm{~T} 15-250$ & $288,367.8$ & $1,006,043.4$ \\
\hline T5.648 & $288,829.2$ & $1,006,054.9$ & $\mathrm{~T} 15.275$ & $288,419.9$ & $1,006,005.5$ \\
\hline T6-5 & $288,973.5$ & $1,006,641.2$ & $\mathrm{~T} 16-25$ & $288,170.9$ & $1,006,069.2$ \\
\hline T6-56 & $288,965.5$ & $1,006,589.2$ & T16-100 & $288,230.7$ & $1,006,025.7$ \\
\hline T6-120 & $288,957.3$ & $1,006,520.1$ & T16-175 & $288,291.3$ & $1,005,981.8$ \\
\hline T6-190 & $288,939.8$ & $1,006,456.9$ & T16-240 & $288,341.4$ & $1,005,945.5$ \\
\hline T6-450 & $288,943.5$ & $1,006,211.1$ & T16-340 & $288,422.8$ & $1,005,887.2$ \\
\hline T6-520 & $288,938.7$ & $1,006,143.2$ & & & \\
\hline
\end{tabular}

